\title{
AI and Robotics in the Fight Against COVID-19 Pandemic
}

\author{
Alaa Khamis ${ }^{1}$, Jun Meng 2 , Jin Wang ${ }^{3}$, Ahmad Taher Azar ${ }^{4}$, Edson Prestes ${ }^{5}$, Howard $\mathrm{Li}^{6}$, \\ Ibrahim A. Hameed ${ }^{7}$ and Tamas Haidegger ${ }^{8,9}$ \\ ${ }^{1}$ General Motors Canada, 500 Wentworth St W, Oshawa, ON L1J 6J2, alaa.khamis@gm.com \\ ${ }^{2}$ College of Electrical Engineering, Zhejiang University, YuQuan Campus,No.38 ZheDa Road, \\ HangzhouZhejiang, 310027, China, junmeng@zju.edu.cn
}

${ }^{3}$ Robotics Institute, Zhejiang University, YuQuan Campus, No.38 ZheDaRoad,Hangzhou 310027, China, dwjcom@zju.edu.cn

${ }^{4}$ Faculty of Computers and Artificial Intelligence, Benha University, Benha,Egypt and College of Computer and Information Sciences, Prince SultanUniversity Riyadh, Saudi Arabia, ahmad.azar@fci.bu.edu.eg, aazar@psu.edu.sa

${ }^{5}$ Informatics Institute, Federal University of Rio Grande do Sul, edson.prestes@ieee.org

${ }^{6}$ Dept. of Electrical and Computer Engineering, University of New Brunswick,Canada, howard@unb.ca

${ }^{7}$ Dept. of ICT and Natural Sciences, Norwegian University of Science andTechnology, Larsg ardsvegen 2, $6009^{\circ}$ Alesund, Norway, ibib@ntnu.no

${ }^{8}$ University Research and Innovation Center (EKIK), Obuda University, Becsi ut 96b, 1034, Budapest, Hungary, haidegger@irob.uni-obuda.hu

${ }^{9}$ Austrian Center for Medical Innovation and Technology (ACMIT),Viktor-Kalpan-str.2., Wiener Neustadt, A-2700, AT, tamas.haidegger@acmit.at

\begin{abstract}
The outbreak of the novel coronavirus and its disease, COVID-19 present an unprecedented challenge for humanity. Artificial Intelligence (AI) and robotics may help fighting COVID-19. Potential applications of AI in this accelerating pandemic include, but are not limited to, early detection and diagnosis, massive agent modeling and simulation, data analytics, assistive robots, disinfection robots, public awareness and patrolling, contactless delivery services, virtual healthcare assistants, drug repurposing and vaccination discovery. This chapter sheds light on the roles AI and robotics can play in fighting this disastrous pandemic, and possible future ones. It highlights several potential applications to transform this challenge into opportunities. This chapter also discusses the ethical implications of AI and robotics during the pandemic and in the postpandemic world.
\end{abstract}




\section{Introduction}

Since the outbreak of the novel coronavirus, the AI and robotics community quickly mobilized and gathered to offer solutions ranging from 3D printed masks ${ }^{1}$ to mass ventilator systems ${ }^{2}$, and to contact tracing and AI driven social distancing apps ${ }^{3}$. Figure 1 shows a non-exhaustive list of reported use of robots worldwide for COVID-19 [1].

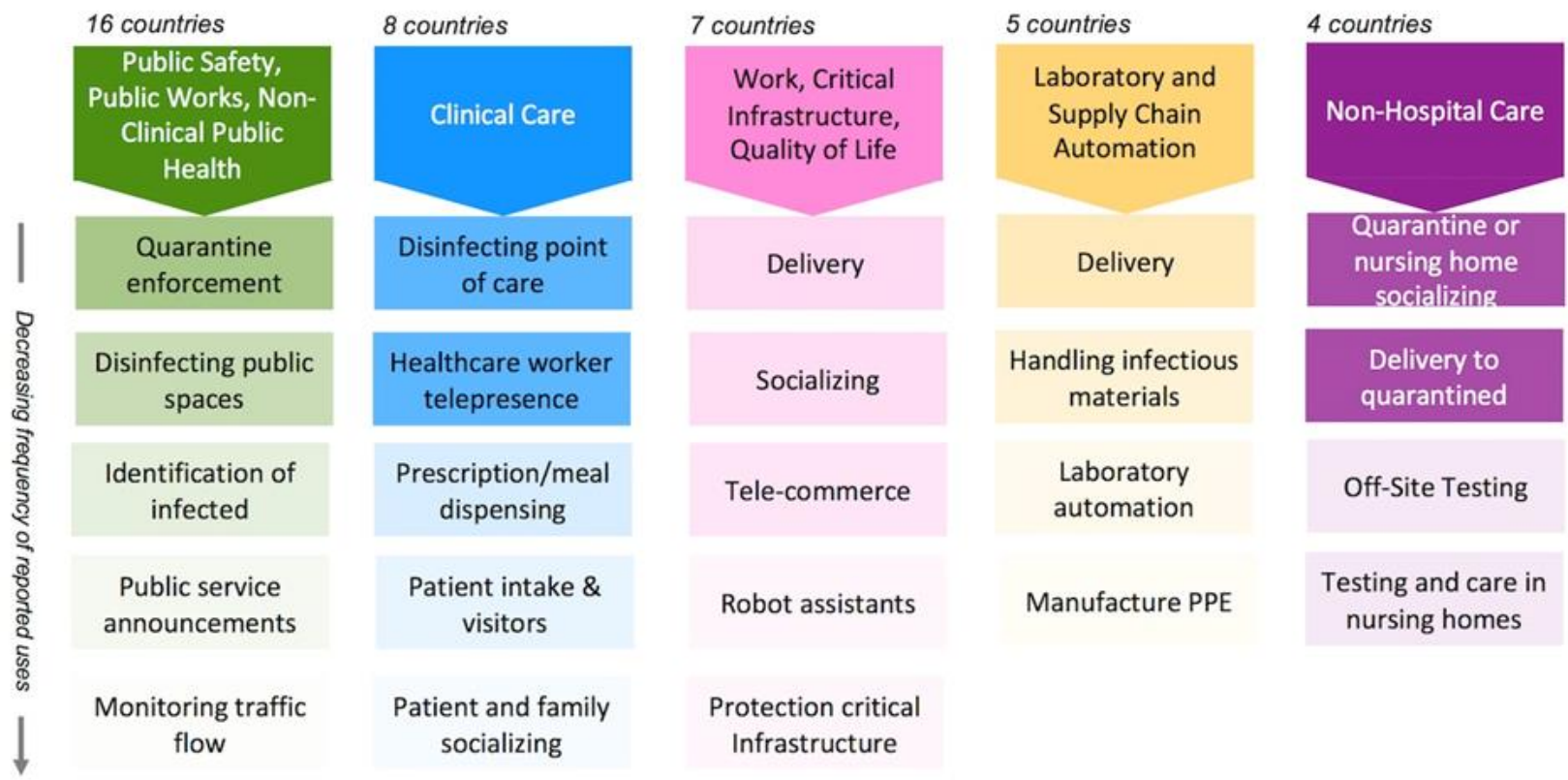

Figure 1 Reported use of robots (Ground, Aerial) worldwide for COVID-19 as of September 2020 [1]

The WHO-China Joint Mission COVID-19 report also cited AI as a key element of the efficient response ${ }^{4}$. The European Commission launched an initiative (managed by the European AI Alliance) to collect ideas about deployable AI and robotics solutions, as well as information on other initiatives that could help solve the ongoing coronavirus crisis: AI-Robotics vs COVID-195. The IEEE is also taking part in the global response; a volunteer steering committee is working on applying the lessons learned to COVID-19 in conjunction with the IEEE Robotics and Automation Society Special Interest Group on Humanitarian Technology ${ }^{6}$. The IEEE Robotics and Automation Society (RAS) is providing a continuous coverage on emerging COVID-19 related technologies via its online platforms ${ }^{7}$.

AI is defined here as an evolving technology that aims at mimicking/reverse-engineering and augmenting biological intelligence to build intelligent systems/processes able to function and interact autonomously within structured/unstructured, static/dynamic and fully/partially

\footnotetext{
${ }^{1}$ https://3dprintingindustry.com/news/3d-printing-community-responds-to-covid-19-and-coronavirus-resources$169143 /$

2 http://massventil.org/

${ }^{3}$ https://www.iit.it/iit-vs-covid-19

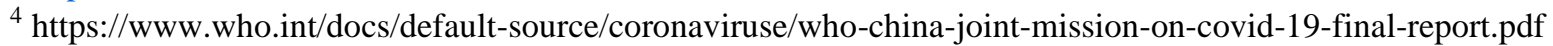

${ }^{5}$ https://ec.europa.eu/digital-single-market/en/news/join-ai-robotics-vs-covid-19-initiative-european-ai-alliance

6 http://roboticsforinfectiousdiseases.org/index.html

${ }^{7}$ https://robohub.org/covid-19-robotics-resources-ideas-for-roboticists-users-and-educators/
} 
observable environments [2]. It encompasses many sub-fields such as perception, knowledge representation, cognitive reasoning, machine learning, data analytics, problem solving, distributed AI and its embodiment, robotics. This chapter describes a number of current and potential future applications of AI and robotics in the battle against the novel coronavirus and its disease COVID19. This chapter is organized as follows. Sections 2 to 10 describe different applications of AI and robotics that a number of countries used during the pandemic such as early detection and diagnosis, massive agent modeling and simulation, data analytics, assistive robots, disinfection robots, public awareness and patrolling, contactless delivery services, virtual healthcare assistants and drug repurposing and vaccination discovery. Section 11 highlights the ethical implications of AI and robotics during the pandemic and in the post-pandemic world. Finally, conclusions are drawn in Section 12.

\section{Early Detection and Diagnosis}

Early detection and early isolation are essential. Detecting and tracking transmission on a large scale in a timely manner, and publishing the data of infected and suspected infections are crucial in prevention and control measures, to minimize further transmission. AI-driven smart wearable medical devices are used in personnel positioning, contact tracing and alarms, and in early warning of areas with potential risks. In China, some anti-epidemic methods focus on early detection and isolation. For example, pandemic drones equipped with temperature and computer vision sensors are used to detect symptoms of infective respiratory disease. Recently, drones are used to monitor temperatures, heart and respiratory levels from a distance up to $10 \mathrm{~m}$ and can detect coughing and sneezing. This can provide researchers with a clear view of infections in public areas and other crowded places such as airports and health care facilities. Detection of body temperature using drones is an effective method for the non-contact detection of body temperature in China. A drone with an infrared thermal imaging lens can take the temperature measurement of each resident in a building without contact and send the data back to the disease control center. Once an abnormal body temperature is detected, medical staff will pinpoint the location of the suspected patient [3]. In addition to human body temperature monitoring, health codes have been widely used since late February in China. Each citizen receives a unique online electronic QR code (see Figure 2) [4].

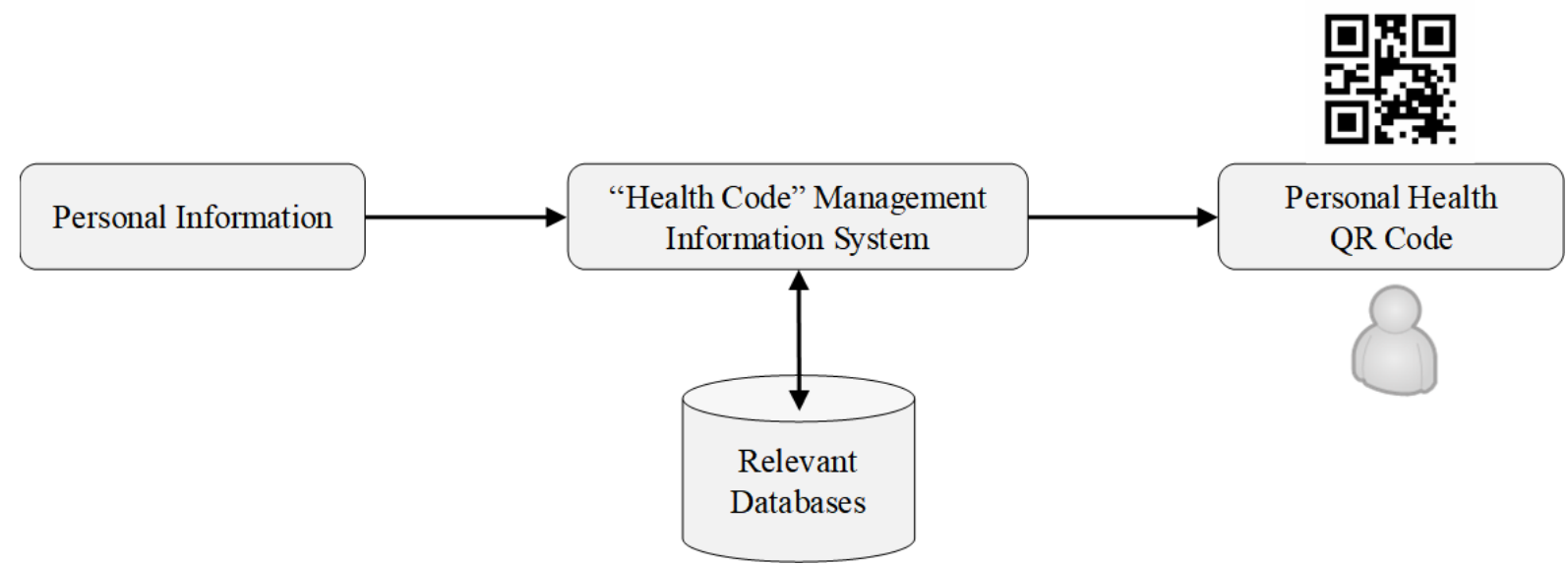

Figure 2 Personal Health QR-based System. The "health code" management information system is based on real data, and connects to relevant databases such as key personnel's dynamic control lists, and issues "health codes" based on the verification and comparison results of the mass declaration information and background data 
AI helmets and AI-powered glasses are used by the Chinese police to identify faces of vehicle occupants and license plates. Alerts are triggered if the vehicle occupant's information can be found in the database of confirmed cases [5]. A smart wearable medical device can be used in an early detection system. This device is used to obtain a person's body temperature, breathing and heartbeat status, whether or not wearing a mask, and update the position information and infection/suspected infection information in real time. At the same time, the precise position of the device and the path of the terminal can be used to calculate a safe and low-risk travel path for people traveling in real time.

Smart wearable medical devices can be useful in the early stage of major infectious disease outbreaks, such as screening population with suspicious physical conditions, quickly performing nucleic acid detection, leading to early detection and isolation. The composition of such a wearable medical device is shown in Figure 3, which is an early concept and prototype developed under the National Nature Science Foundation of China (NSFC) program No. 7204102194.

The three major functions of this wearable devices-based system are:

1. Outbreak Visualization: based on individual body temperature charts, spatiotemporal dynamics of personnel mobility, people gathering heatmap, spatiotemporal map of confirmed cases.

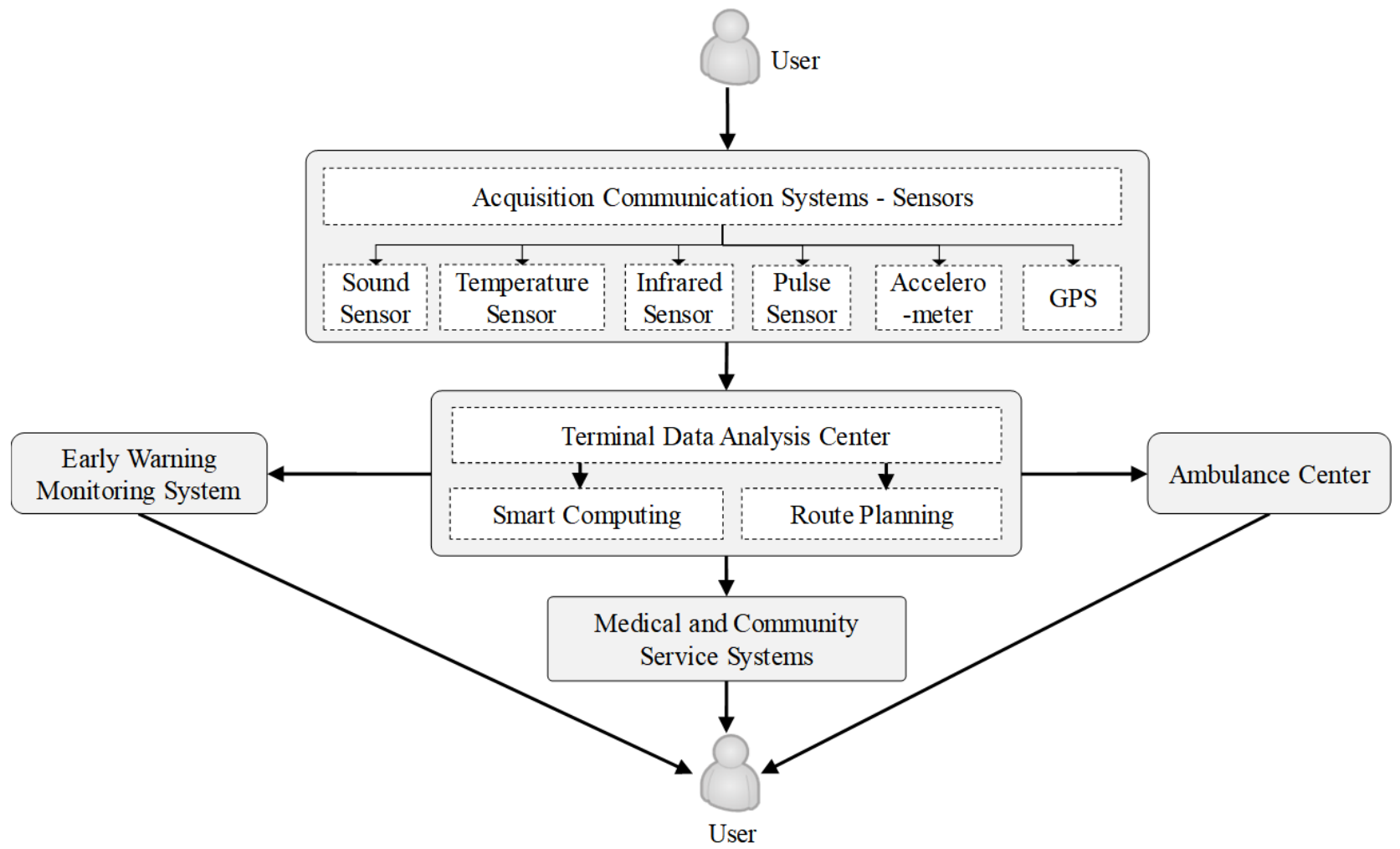

Figure 3 The composition of smart medical wearable devices, which can real-time grasp each person's current body temperature, heartbeat, breathing, confirmed infectious disease status, whether they are in contact with a confirmed/suspected infectious case, whether a mask is worn during going out, the epidemic situation during the period and so on. This information is uploaded to the terminal in real time to generate a spatiotemporal map of the epidemic situation, which quickly, multi-dimensionally and comprehensively displays the current body temperature, breathing, and heartbeat information of the personnel. 
2. Early Detection and Early Warning: screen out suspicious cases in due time.

3. Pinpoint and Route Planning: propose safe travel paths for travelers.

In the early stage of major infectious diseases, people with suspicious physical conditions detected by the smart wearable medical devices will be screened timely for further CT examinations or nucleic acid tests. There are some data-driven approaches using artificial intelligence to diagnose patients through chest scans. Li and Qin, et al [6] proposed a threedimensional deep learning framework to detect COVID-19 using chest CT, named COVID-19 detection neutral network (COVNet). University of Waterloo's VIP Lab develops a COVID-Net: a convolutional neural network for COVID-19 detection via chest radiography $(13,800$ chest XRay of 13,725 cases from around the world ${ }^{8}$. In China, there is a supercomputer that provides doctors around the world with free access to an artificial intelligence diagnostic tool for early identification of Covid-19 patients based on a chest scan. The AI system on the Tianhe-1 computer can go through hundreds of images generated by computed tomography (CT) and give the diagnosis in about 10 seconds ${ }^{9}$. What more, Wang et al [7] propose an innovative method that does not require specialized medical imaging equipment and uses footage from Kinect depth cameras to identify respiratory patterns of patients. They first apply a GRU neural network with bidirectional and attentional mechanisms (BI-AT-GRU) to classify six clinically significant respiratory patterns.

\section{Massive Agent Modeling and Simulation}

Model prediction is to better understand the spatiotemporal transmission path of major infectious diseases, to analyze the spatiotemporal transmission data of infectious diseases, to timely prevent and control infectious diseases, and to analyze the risks of various policies and measures during the epidemic. The system described in the previous section combines smart wearable medical devices with infectious disease prevention and control. It also combines data in geographic information systems to construct a multi-dimensional, multi-scale infectious disease spatiotemporal framework, and establish a model for the spatiotemporal spread of infectious diseases. The system can be used to analyze the spatiotemporal spread of infectious diseases from various perspectives, such as the natural meteorological environment, land use, and population density, etc. The cellular automata models are used to simulate various countries and regions. Various epidemic prevention policies and measures are used to control the spread of infectious diseases and predict major infectious diseases. The risks of various prevention and control policies and measures during the current period provide theoretical basis for the prevention and monitoring of future infectious diseases.

Using the data collected by wearable devices and the GIS technology, the temporal and spatial transmission characteristics of infectious diseases are correctly described. First, data mining is used to analyze the external environment that affects the occurrence and spread of infectious diseases, including regional economic conditions, regional population density, regional ecological environment and other external factors. Through geographic information and spatial analysis,

\footnotetext{
${ }^{8}$ https://medium.com/@ sheldon.fernandez/covid-net-an-open-source-neural-network-for-covid-19-detection$48 \mathrm{~b} 8 \mathrm{a} 55 \mathrm{e} 6 \mathrm{~d} 44$

${ }^{9}$ https://www.thestar.com.my/tech/tech-news/2020/03/16/covid-19-chinese-supercomputer-uses-artificialintelligence-to-diagnose-patients-from-chest-scans
} 
spatial trends are further analyzed, and statistical methods are used to determine the factors and their effects. Combined with the data obtained from smart wearable medical devices, a spatiotemporal spread model of infectious diseases is constructed, and the infectious disease prediction model is obtained. Figure 4 is a spatiotemporal modeling flowchart of infectious disease transmission based on the GIS technology and wearable device data.

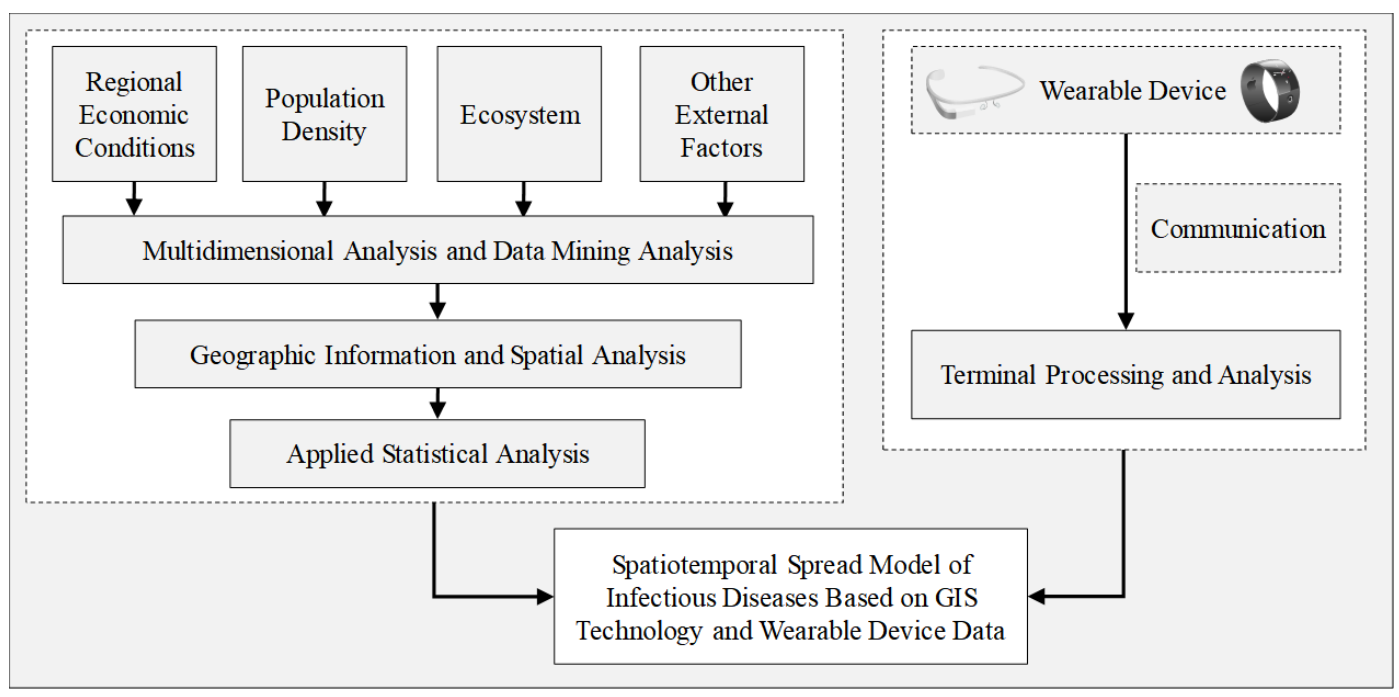

Figure 4 Spatiotemporal modeling flowchart of infectious disease transmission based on GIS technology and wearable device data.

With the spatiotemporal spread model of infectious diseases, the GIS technology and wearable device data, external factors related to the spread of infectious diseases are determined. The cellular automaton is used to model and simulate the spatiotemporal spread of infectious diseases. By choosing various external factors, the spatiotemporal spread of infectious diseases is simulated, and the risks under certain measures and policies are calculated and predicted. The simulation results of the spread of infectious diseases in different countries and regions are simulated. For example, after setting the population and geographical conditions of different countries/regions and simulating the evolution of different epidemic prevention measures, the spread of infectious diseases in different countries/regions can be predicted. Suggestions can be made for different countries/regions to adopt appropriate epidemic prevention measures. Figure 5 is a flowchart of the spread of infectious diseases based on cellular automata.

This system implements real-time early warning based on the following decision rules.

1. Using wearable devices, when two people visit the same place at the same time, if one of them becomes a confirmed case, the other person will be marked as a suspected case.

2. If a person has never cross path with a confirmed infected person or suspected case, this person is considered not infected.

3. If a person may encounter a confirmed or suspected case when if he travels, the wearable device will advise him and prevent him from coming into contact with the confirmed case or suspected case. 


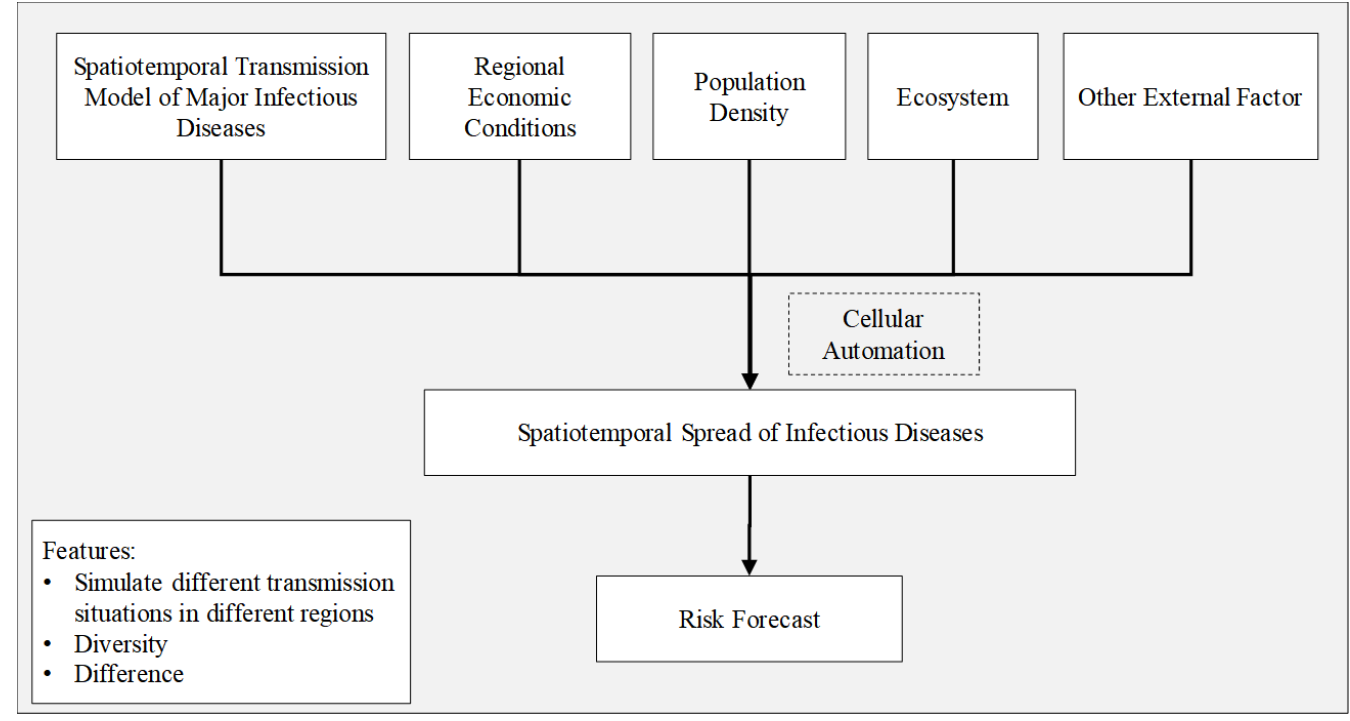

Figure 5 Modeling and simulation flowchart of spatiotemporal transmission of infectious diseases based on cellular automaton

4. Wearable devices can accurately locate the infected person. Once the infected person leaves, disinfection can be conducted immediately.

\section{Data Analytics}

AI-powered data analytics provides the ability to discover, recognize and predict complex patterns and trends in different types of data at different levels of abstractions. During the pandemic of SARS-CoV-2, multimodal data is collected by robots, cell phones and wearable devices. This data can be archived/steaming/live data, structured/unstructured data, numerical/non-numerical (text, image, audio, and video) and multimodal data. Examples of the data include, but are not limited to, body temperature, heart rate, breathing rate, $\mathrm{SpO}$, blood pressure, acoustic data, accelerometer data, ultrasound, computerized tomography (CT) scans/chest radiography, depth camera data and mobility data, to name just a few.

Insights are the new gold not the data, since data is worth very little unless the insights are understood. In the context of coronavirus and COVID-19 pandemic, the insights play a crucial role in the understanding, predicting, and decision-making processes. Researchers use four main types of data analytics, namely, descriptive analytics, predictive analytics, diagnostic analytics, and prescriptive analytics as illustrated in Figure 6 [8]. Descriptive data analytics provides insight into the past and the present while predictive analytics forecasts the future. Diagnostic analytics provides root-cause analysis and perspective analytics advises on possible outcomes and their anticipated impacts. 


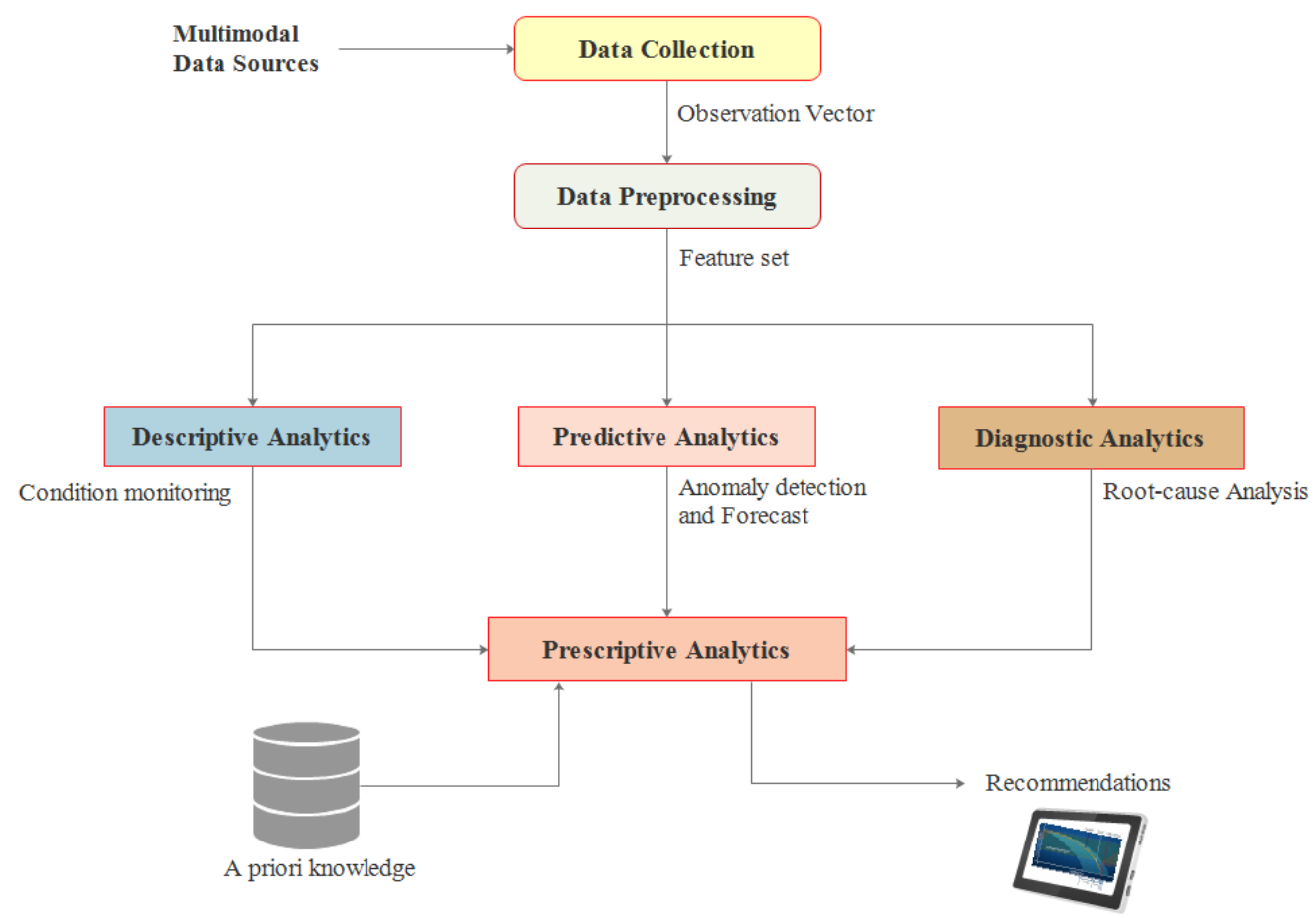

Figure 6 Data Analytics

Descriptive data analytics provides a better understanding of the data and its nature and identifies patterns or relationships in the data. It provides insight into the past and the present by answering questions like what has happened? what happens now? what is the trend of a certain variable? what is the relationship between variables? and how an item is performing with respect to other items or the benchmark? Various web-based tools and mobile apps provide different forms of descriptive analytics for COVID-19 such as the Johns Hopkins coronavirus resource center ${ }^{\frac{10}{}}$, worldometer $^{\underline{11}}$, covidvisualizer $\frac{12}{}$ and DOMO coronavirus tracker $\frac{13}{}$. These tools summarize and visualize descriptive statistics related to the number of confirmed COVID-19 cases in every country, total number of deaths, number of recovered patients, number of active causes, number of recovered cases, etc. This publicly available information helps achieving public health measures advocated by WHO. Several wearable devices with positioning are used in China and other countries to monitor coronavirus patients, trace doctors, nurses and other clinicians who have contracted the disease as quickly as possible and monitor the pandemic spread across the country. Some tools were developed to facilitate contact tracing (Coronamap ${ }^{14}$ ), search for masks (Maskmap ${ }^{15}$ ), visualize the disease transmission ${ }^{16}$ and show how the community is responding differently due to COVID-19 $\frac{17}{}$. Apple and Google are building a coronavirus tracking system into iOS and Android allows users to share data through Bluetooth Low Energy (BLE) transmissions

\footnotetext{
${ }^{10}$ https://coronavirus.jhu.edu/map.html

11 https://www.worldometers.info/coronavirus/

12 https://www.covidvisualizer.com/

13 https://www.domo.com/coronavirus-tracking

14 http://coronamap.site/

15 https://maskmap.site/

16 https://www.gisaid.org/epiflu-applications/next-hcov-19-app/

17 https://www.google.com/covid19/mobility/
} 
and approved apps from health organizations. Based on this system, Covid Watch $\frac{18}{}$ contact trucing app is developed to empower people and protect their communities from COVID-19 using an anonymous exposure notification system. Other descriptive analytics tools are developed as software-as-service ${ }^{19}$ for contact tracing, locating and conceptualizing infectious disease spread by incorporating hundreds of thousands of multimodal data sources such as statements from official public health organizations, digital media, global airline ticketing data, livestock health reports, population demographics, etc.

Predictive models make prediction about future values of data and forecasts new proprieties instead of just exploring data properties like in case of descriptive analytics. These models answer questions like what would happen?; when would it happen? and where would it happen?. Despite the availability of several descriptive analytics tools during the pandemic, there are much less predictive models. Predicting future states using hand-crafted mathematical models or using datadriven approaches depends on a number of factors such as targeted prediction (exact values, reasonable range, trend, seasonality, etc.), type of data (stationary versus non-stationarity), desired forecast window and availability of a priori knowledge. Generally speaking, predicting exact values of non-stationary variables for an extended forecast window is challenging unless there is enough multimodal data and a priori knowledge about the variables of interest. For example, COVID-19 cases cannot be accurately predicted using only past and current case counts reported by WHO and applying oversimplified data mining algorithms such as moving average, auto regressive moving average, logistic regression, etc. Infectious disease models or epidemiological models that incorporate transmission dynamics and clinical dynamics should be considered. Existing infectious disease predictive models are oversimplified or a repurposed version of similar disease models assuming that the novel corona virus behaves like influenza or SARS-CoV. These models cannot be fully trusted to make decisions. This is mainly due to lack of enough information about the novel coronavirus and COVID-19 such as self-mutation capability of the virus, incubation period, how it spreads, what are all the different ways the virus can be transferred between people? what is the transmission time or how long does it take to transmit the virus to other person from the moment the virus enters a person's body?, how temperature and humidity can impact virus transmission, a priori knowledge and demographic information like age, gender, blood type, other diseases, previous vaccination record, etc.. For example, epidemic calculator $\underline{20}$ implements a classical infectious disease model - SEIR (Susceptible $\rightarrow$ Exposed $\rightarrow$ Infected $\rightarrow$ Removed) [9,10]. This model uses different transmission dynamics and clinical dynamics parameters such as population size, number of initial infections, transmission times, case fatality rate, hospitalization rate, etc. to simulate the disease's progression. Robert Koch Institut 21 developed a predictive model based on not only current case counts of WHO but also using a novel epidemiological model that integrates the effect of population behavior changes due to government measures and social distancing. This predictive model can provide 6-day forecasts of COVID-19 case counts by country. The predictive model of Institute for Health Metrics and Evaluation ${ }^{22}$ at the University of Washington has a wide range of projections for deaths from COVID-19 based on different underlying assumptions and how they change, such as the effect of social distancing or widespread testing. Due to lack of enough data, trustable predictive models need to focus on

\footnotetext{
${ }^{18} \mathrm{https://www.covid-watch.org/}$

$19 \mathrm{https://bluedot.global/}$

20 http://gabgoh.github.io/COVID/

21 http://rocs.hu-berlin.de/corona/docs/forecast/results_by_country/

22 https://covid19.healthdata.org/
} 
predicting trends and reasonable ranges, rather than exact numbers. As we collect more unbiased and consistent data and gain more information about this novel virus, these predictive models will get better and more trustworthy.

Diagnostic analytics provides root-cause analysis and answers questions such as why did a health-related problem happen? or why would a problem happen? Finally, a prescriptive analytics model will process information about the current condition of the patient or the population generated by descriptive analytics module, anomaly detection and forecast from predictive analytics module, root-cause analysis generated by the diagnostic model and any other a priori knowledge that may be available about the patient medical records and/or places recently visited. It then produces timely recommendations for proactive actions or intervention plans to mitigate any possible risk. This decision support or recommendation engine answers the following questions, how would the predictions obtained from predictive models impact everything else? what are the proactive decision/actions to be made? how we benefit from predictions/recommendations? what are the best actions to make? what is the best time to take this action? and what would be the impact of this action? Based on the insights generated from the descriptive and predictive analytics models, different strategies to flatten the outbreak curve, end the pandemic and bring life to normal are recommended. For example, researchers at Imperial College concluded that there are essentially three ways to end the pandemic, which are vaccination, herd immunity or permanently change our behavior/society [11]. The consequences of doing nothing, mitigation and the hammer \& dance strategies were explained in [12].

\section{Assistive Robots}

Robots can perform many assistive tasks during the pandemic to mitigate the risk to healthcare professionals. Applications of assistive robots include, but are not limited to, medical care, nursing, patient monitoring, performing lab work, cooking and serving medication, meal delivery to patients in isolation wards. Several assistive telecare robots, community medical robots and telemedicine services are used during the outbreak of the novel coronavirus. For example, doctors use the mounted iPad on Spot to remotely interact with coronavirus patients, ask them question in order to assess the patients ${ }^{23}$. Tommy helped Italian medical teams in treating COVID-19 patients $^{24}$ (Figure 7).

Saskatchewan Remote Presence Robots (Figure 8) are used to deliver health care to rural and remote regions of Saskatchewan, Canada in order to protect physicians on the front lines fighting the COVID-19 pandemic ${ }^{25}$.

The system illustrated in Figure 9 uses artificial intelligence, big data, robots, the Internet of Things, modern medical technology, cloud computing and other platforms and methods. According to the users and scenarios, it can be divided into three parts, namely, community robots, the monitoring data platform and the diagnostic platform [13].

Each community entrance can be equipped with several robots, and the position of each robot can be accurately obtained. For each person entering and leaving the community, the identification is verified (for example, fingerprint identification, face recognition, biometric identification), the

\footnotetext{
${ }^{23}$ https://www.telegraph.co.uk/technology/2020/04/23/robot-dogs-used-help-doctors-remotely-assess-coronaviruspatients/

${ }^{24} \mathrm{https}$ //www.pri.org/stories/2020-04-08/tommy-robot-nurse-helps-italian-doctors-care-covid-19-patients

25 https://thestarphoenix.com/news/local-news/sask-ready-to-deploy-medical-robots-to-help-fight-against-covid-19/
} 
body temperature is collected, the blood pressure is monitored, the blood test can be performed to determine whether a face mask is necessary. For suspected cases, one can further use the pneumonia test kit on the community robot and take pictures of the corresponding disease location. At the same time, the medical staff connected to the remote diagnosis platform through the voice dialogue system can communicate quickly. The robot will upload information about the suspected patient to the monitoring data platform. The robot will record and send data to the monitoring personnel. The robot can self-sterilize at regular intervals and sterilize the air in the surrounding environment and effectively prevent and reduce cross-infection.

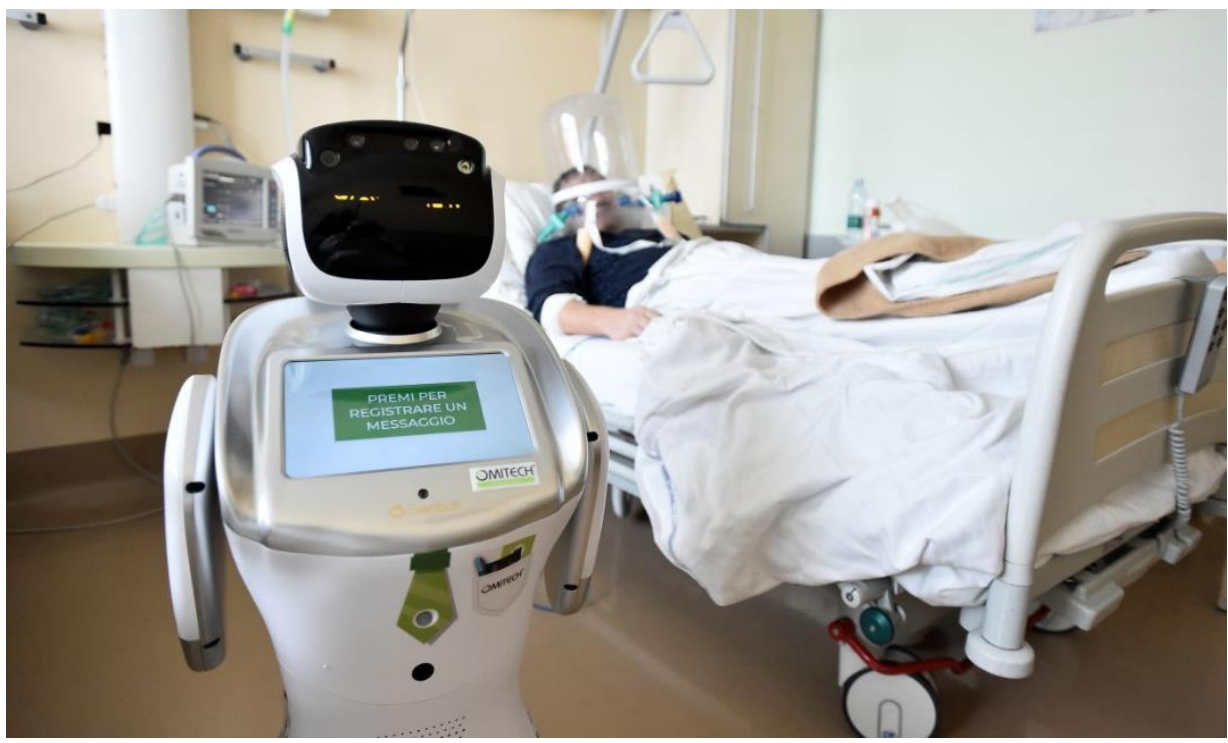

Figure 7 Tommy, the robot nurse. Credit: Flavio Lo Scalzo/Reuters

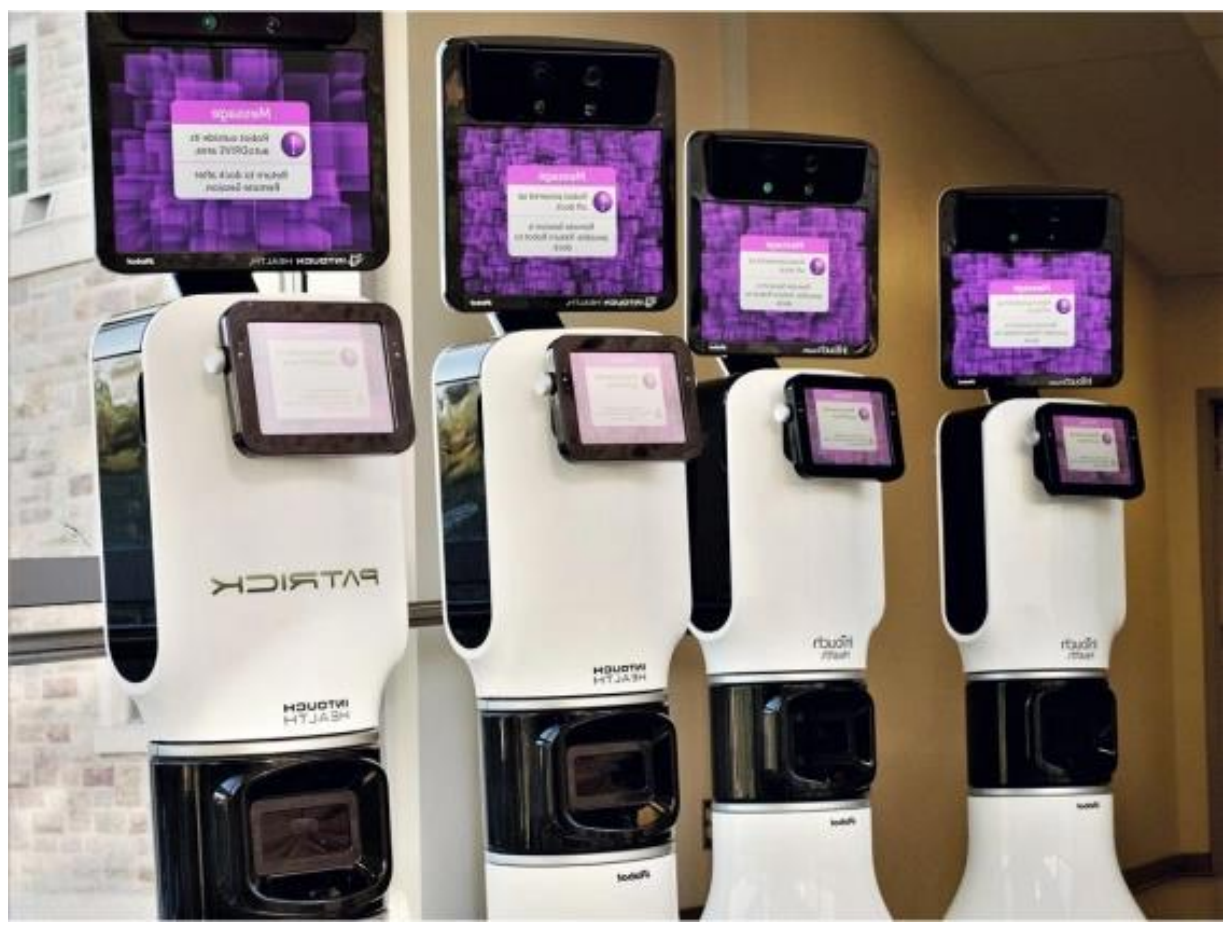

Figure 8 Saskatchewan Remote Presence Robots. Credit: University of Saskatchewan and Saskatchewan Health Authority Remote Presence Robotic Program 


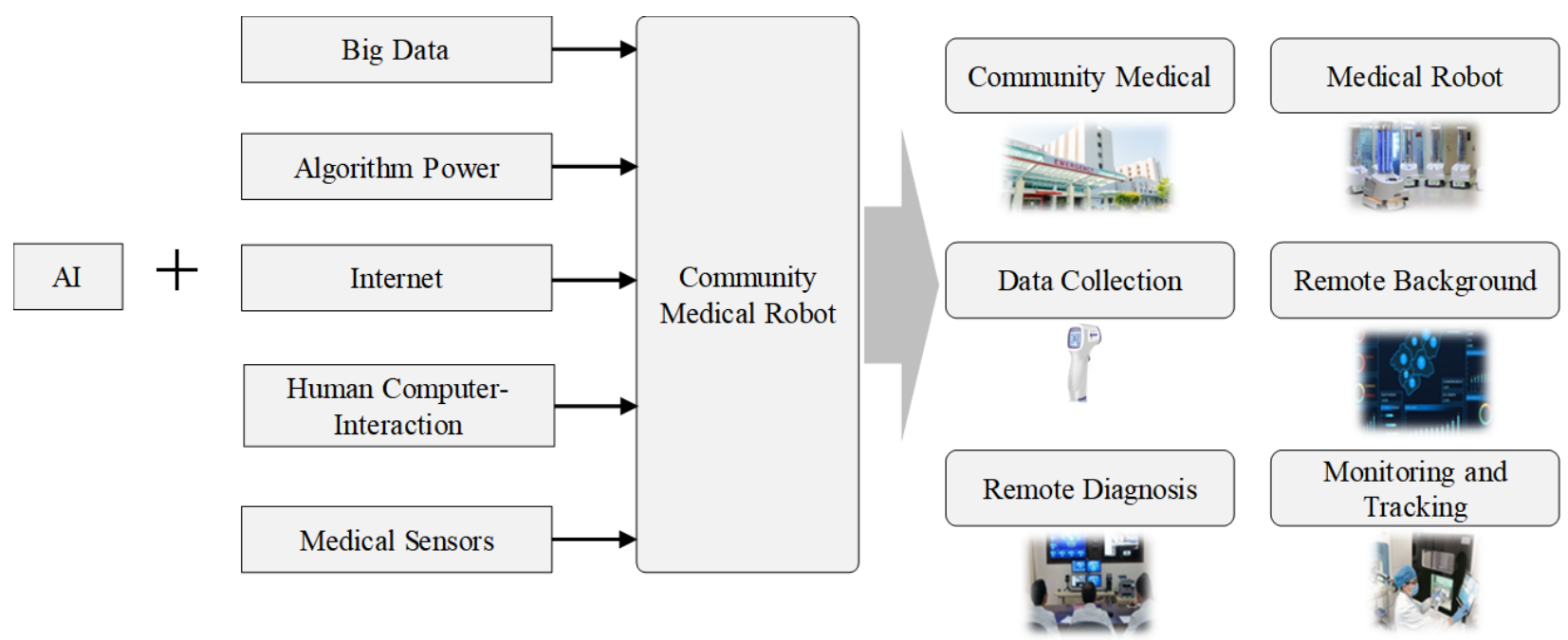

Figure 9 A composition framework diagram of community medical robots

The monitoring data platform collects information uploaded by medical robots in various communities and stores data (time and location of examination for suspected cases). Data mining (mines potential cases based on recorded data such as temperature, reagents, pulse, blood routine, location, etc.), data analysis and calculation (statistical analysis of the epidemic of the entire region) are performed. Forecasts of the region in the next few days are given. Timely feedback is given to the epidemic units and personnel to facilitate corresponding control decisions.

The diagnostic platform obtains real-time information through the monitoring data platform. The information is mainly related to the disease. At the same time, it can make video and voice calls with the suspects detected by the community robot. It can also check and analyze the photos of the suspects in order to provide medical care.

The Chinese Academy of Sciences developed a robot to remotely collect oropharyngeal swabs from patients for testing, so the medical workers do not have to directly swab the patients ${ }^{26}$. Figure 10 shows the three major systems of community medical robots.

Some more simplistic solutions (e.g., RoboAds) include only a mobile robot platform for autonomous positioning of kiosks on the one hand to reach the target audience with interactive (touchless) billboards, while taking telemetry and collecting data on people's health condition (primarily body temperature).

\section{Disinfection Robots}

Disinfecting surfaces and the environment, performed routinely in hospitals, has always been essential for infection prevention and control. Beyond regular surface wiping with highly efficient detergents, a key strategy has been to allow the active ingredient to spread to all areas of the room, however this also needs complete lockdown and heavy air ventilation. UV-C irradiation (200$280 \mathrm{~nm}$ ) is also an efficient disinfectant, if the radiation reaches all critical surfaces. Robots are

\footnotetext{
${ }^{26}$ https://www.straitstimes.com/asia/east-asia/coronavirus-china-develops-robot-for-throat-swab-sampling
} 
considered as an effective technology for disinfection, preventing any harm to humans. There are two major types of applications: indoor and outdoor.

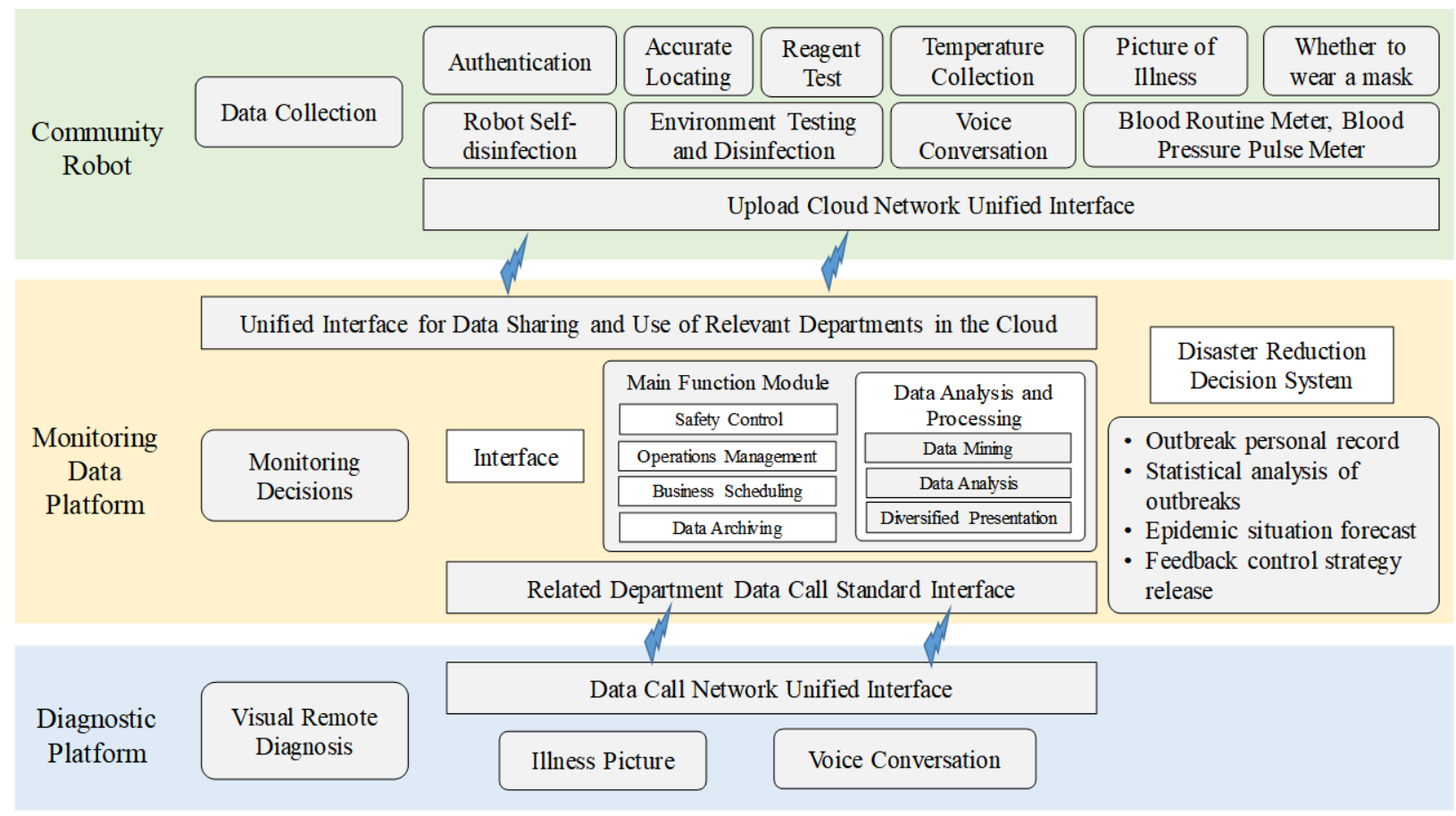

Figure 10 Community Medical Robots

- Outdoor Disinfection usually means the spraying of an active material ( $\mathrm{NaClO}$, sodium hypochlorite in a very low concentrate). While scientists argue that this method has very limited effect on the spread of a virus [14], engineers have developed both teleoperated and autonomous ground and aerial vehicles (Figure 11). Several drone manufacturers have modified their agricultural models in order to spray disinfectant over large areas ${ }^{27}$. These disinfecting drones have been used for the first time in Europe, and have been deployed in China, Chile, Indonesia, the Philippines, Colombia and the United Arab Emirates. XAG Robot has deployed
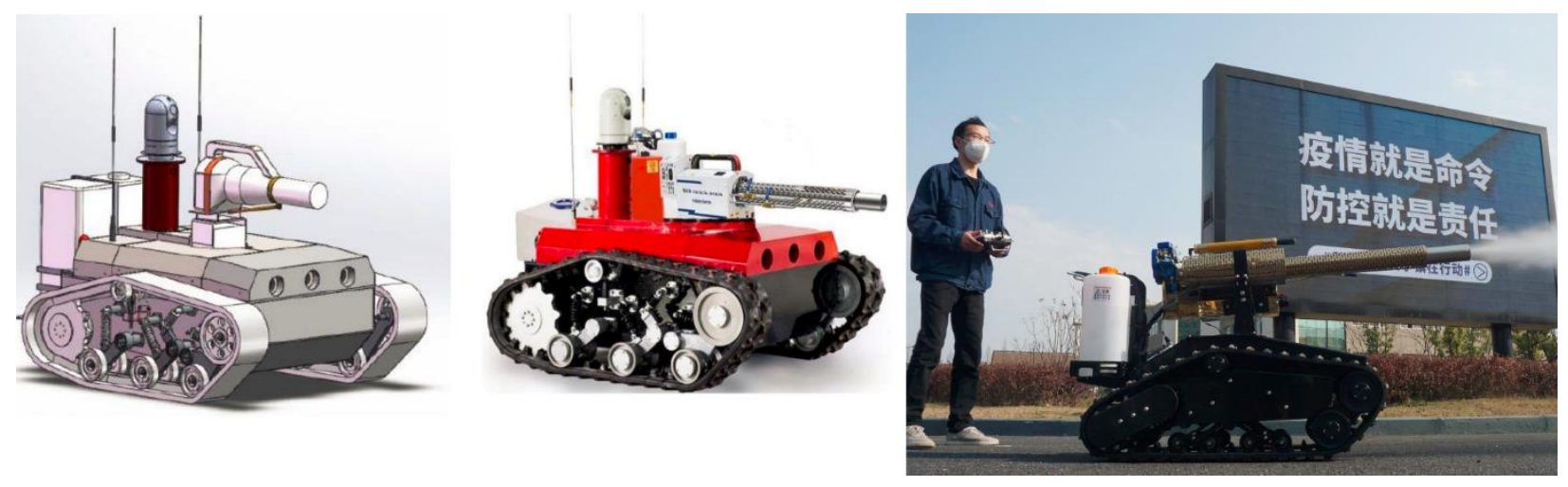

Figure 11 Outdoor disinfecting robot from Siemens, the Chinese company Aucma working in the joint laboratory for robot applications in Qingdao. The robot was designed using CAD and the actual system was built in just a week. Credit: SIPA Asia

${ }^{27}$ https://cadth.ca/robotic-systems-disinfecting-surfaces-hospital-rooms-and-other-health-care-environments 
disinfectant-spraying robots and drones in Guangzhou, China ${ }^{28}$. Nevertheless, safety of such systems remains a major concern $^{29}$, while novel control algorithms greatly support service robot applications [15].

- Indoor Cleaning robots are available in the market for ward disinfection. The LightStrike Germ-Zapping robots (XENEX, San Antonio, TX) became the first disinfection system of its kind to deliver intense germicidal action from pulsed UV-C at 200-315 $\mathrm{nm}^{30}$ over 12 years ago. The Danish UVD Robots ApS was founded in 2016 by Blue Ocean Robotics and its solution offers indoor mobility to deliver the UV-C irradiation ${ }^{31}$. It was the recipient of the IEEE/IFR 2019 Innovation and Entrepreneurship Award (IERA) ${ }^{32}$ (Figure 12). Shenzhen-based YouiBot was already making autonomous robots, and quickly equipped its existing mobile robot base with thermal cameras and UV-C emitting bulbs ${ }^{33}$. Akara Robotics, a spin-off from Trinity College Dublin is testing and producing a new autonomous mobile robot that is designed primarily to sterilize a room with UV light. The Indian Milagrow offers its Indoor Disinfection RoboCop. The Turkish Milvus Robotics calls its UV disinfecting robot SEIT-UV, with $360^{\circ}$ disinfecting coverage, software and sensor-based safety features. The Chinese WellWit Robotics offers its Pulsed Light Germ-Killing Robot (WDR01A). The PBA Group announced the rollout of their Sunburst UV Bots, UV-disinfecting autonomous mobile robots together with Singapore based Frasers Property Group. A similar solution is developed by Anscer Robotics in Bangalore, India ${ }^{34}$. TIAGo robot equipped with UV-C sources can be used for cleaning and disinfection at hospitals and other public areas ${ }^{35}$. A recent development from Rovenso targets directly offices and commercial spaces with its focused UV$\mathrm{C}$ disinfectant robot $^{36}$.

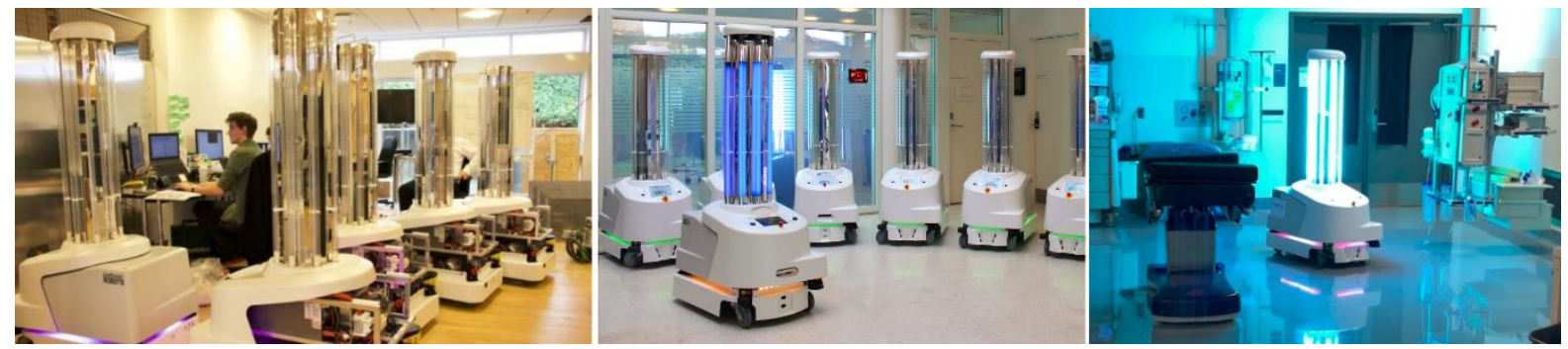

Figure 12 Indoor disinfecting robot UVD, consisting of a mobile base equipped with multiple LIDAR sensors and an array of UV lamps mounted on top. Credit: UVD Robots.

\section{Public Awareness and Patrolling}

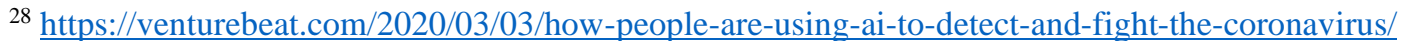

${ }^{29} \mathrm{https} / / / \mathrm{www} . w e f o r u m . o r g / a g e n d a / 2020 / 03 /$ three-ways-china-is-using-drones-to-fight-coronavirus/

30 https://www.xenex.com/our-solution/lightstrike/

31 http://www.uvd-robots.com/

32 https://spectrum.ieee.org/automaton/robotics/medical-robots/autonomous-robots-are-helping-kill-coronavirus-inhospitals

${ }^{33}$ https://www.bbc.com/news/business-51914722

34 https://mobilerobotguide.com/2020/04/01/robotic-solutions-for-covid-19/

$35 \mathrm{http} / / / \mathrm{blog}$.pal-robotics.com/how-to-build-a-solution-for-fighting-coronavirus-using-the-tiago-base-robot/

$36 \mathrm{https://spectrum.ieee.org/automaton/robotics/industrial-robots/rovenso-uv-disinfection-robot}$
} 
Drones have been widely used for the fight against COVID-19. Drones can be equipped with high-power speakers at large gatherings. Warnings are broadcasted to the crowd. Drones can be used to identify people not wearing masks. People are reminded by drones to wear masks. At the same time, drones can detect people quickly through thermal imaging and target recognition systems. Naturally, mobile health solutions have been widely combined with such robotic solutions [16].

Another example is Boston Dynamics' Spot that is used at parks to remind visitors of social distancing measures ${ }^{37}$. Speech assistants are used for home isolation and control. For residents who need to be isolated at home, the speech assistants will provide appropriate advices. For example, "It is recommended that you and your family undergo a 14-day medical observation at home. We will submit your information to the neighborhood and community health service center. The center will collect further information later. If you need any help, please contact the your community center in time. Thank you!"38. Another example is Knightscope Robotics ${ }^{39}$ that can be used for patrolling corporate facilities. The robots are used for remote monitoring of streets and corporate campuses (Figure 13).

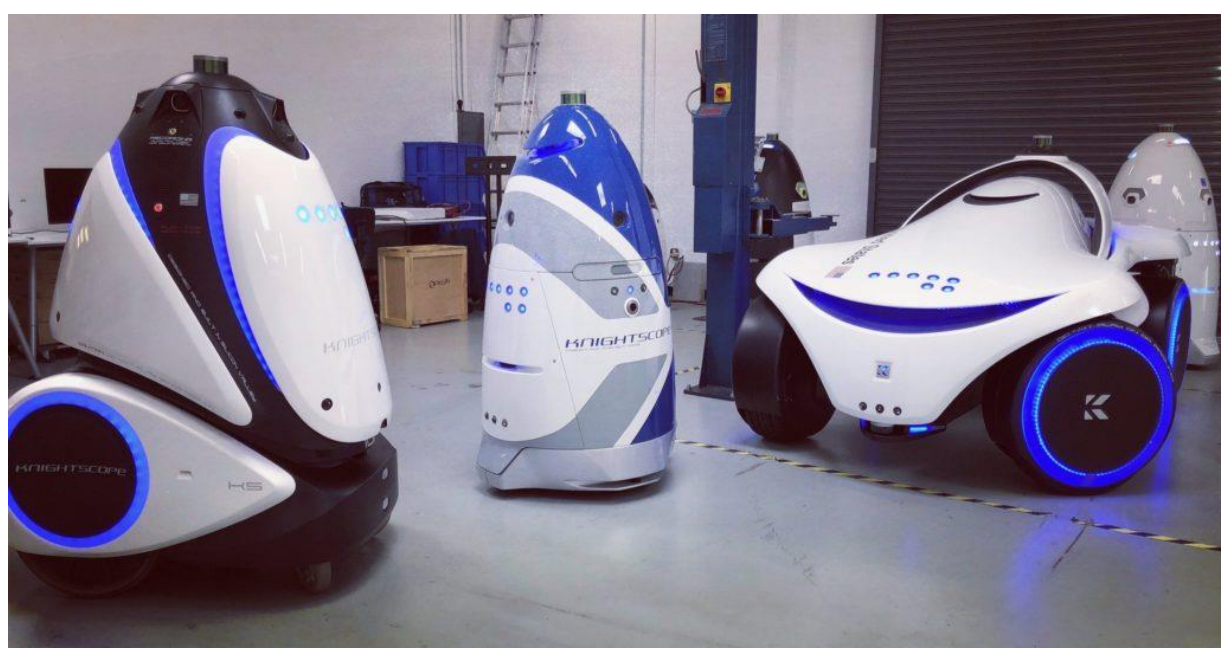

Figure 13 The Knightscope lineup of indoor and outdoor telepresence robots. Credit: Knightscope Robotics

\section{Contactless Last-mile Delivery Services}

Fighting the coronavirus pandemic demands last-mile delivery robots. Last mile is a term used in supply chain management and transportation planning to describe the movement of people and goods from a transportation hub to the destination [17]. Various innovative solutions for last- mile delivery are currently being developed or tested to reduce the delivery cost, increase customer satisfaction and minimize the negative environmental impact. These solutions include, but are not limited to, cargo-bikes, semi and fully autonomous last-mile delivery, delivery Droids (Bots), EPlaette, Postal delivery, driverless deliveries and privately-owned AV. According to Makinsey, semi and fully autonomous last-mile delivery will reduce costs by approximately $10-40 \%$ [18].

\footnotetext{
${ }^{37}$ https://www.straitstimes.com/singapore/robot-reminds-visitors-about-safe-distancing-measures-in-bishan-ang-mokio-park

38 https://www.atlantis-press.com/article/25881703.pdf

39 https://mobilerobotguide.com/2018/07/20/knightscope/
} 
The highly contagiousness coronavirus results in one of the worst outbreaks. Social distancing is the main measure taken to reduce the spread of the virus through minimizing the contact between people. However, frontline workers who treat the patients or deliver medicine or food for them and even people at home who order essential goods like medication or grocery are still at risk. Contactless last-mile delivery systems and services can result in avoiding physical contact between caregivers and patients or between delivery workers and the recipients. These contactless delivery systems benefit from the rapid proliferation of connected technologies and the recent advancements in semi and fully autonomous delivery platforms which revolutionize the urban logistics and provide a safe and efficient delivery methods for medical supplies [19], medications [20], food, grocery and other goods [21,22]. Figure 14 shows AIMBOT ${ }^{40}$, an autonomous mobile robot developed by UBTECH Robotics, performs disinfection tasks at Shenzhen Third Hospital.

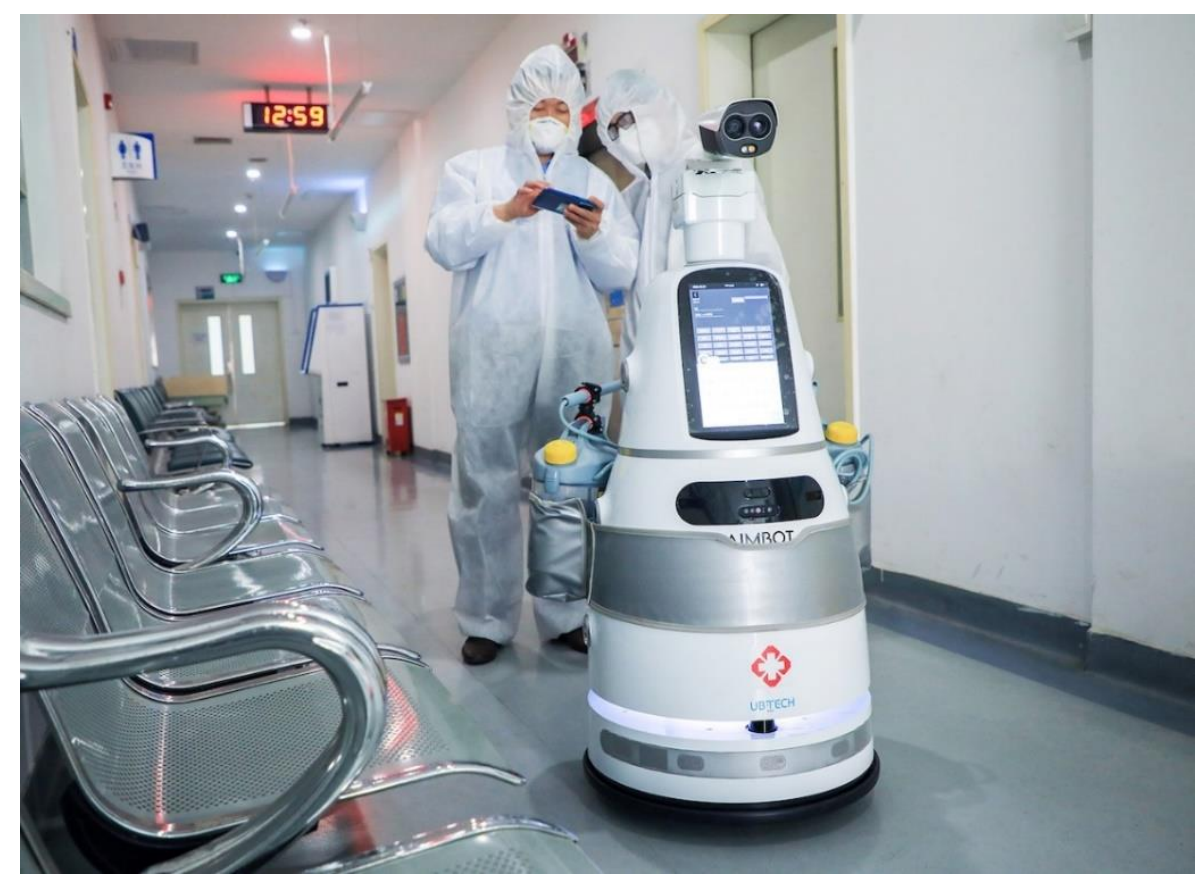

Figure 14 AIMBOT, an autonomous mobile robot. Credit: UBTECH Robotics

The demand for food delivery facilities has never been higher as most restaurants and cafés are closed and the public are advised to stay at home to prevent the virus from spreading. However, despite COVID-19's incredibly infectious nature, more and more suppliers prefer contactless distribution solutions to reduce the risk to consumers and the delivery personnel. For example, Starship ${ }^{41}$ has hundreds of robots delivering food to customers. Drones are an ideal solution for contactless food delivery to remote areas. Figure 15 shows Nuro driverless vehicle that has been approved for delivery tests in California in April 2020 [22].

\footnotetext{
${ }^{40} \mathrm{https} / / / \mathrm{www} . w e f o r u m . o r g / a g e n d a / 2020 / 03 /$ three-ways-china-is-using-drones-to-fight-coronavirus/

${ }^{41}$ https://www.starship.xyz/
} 


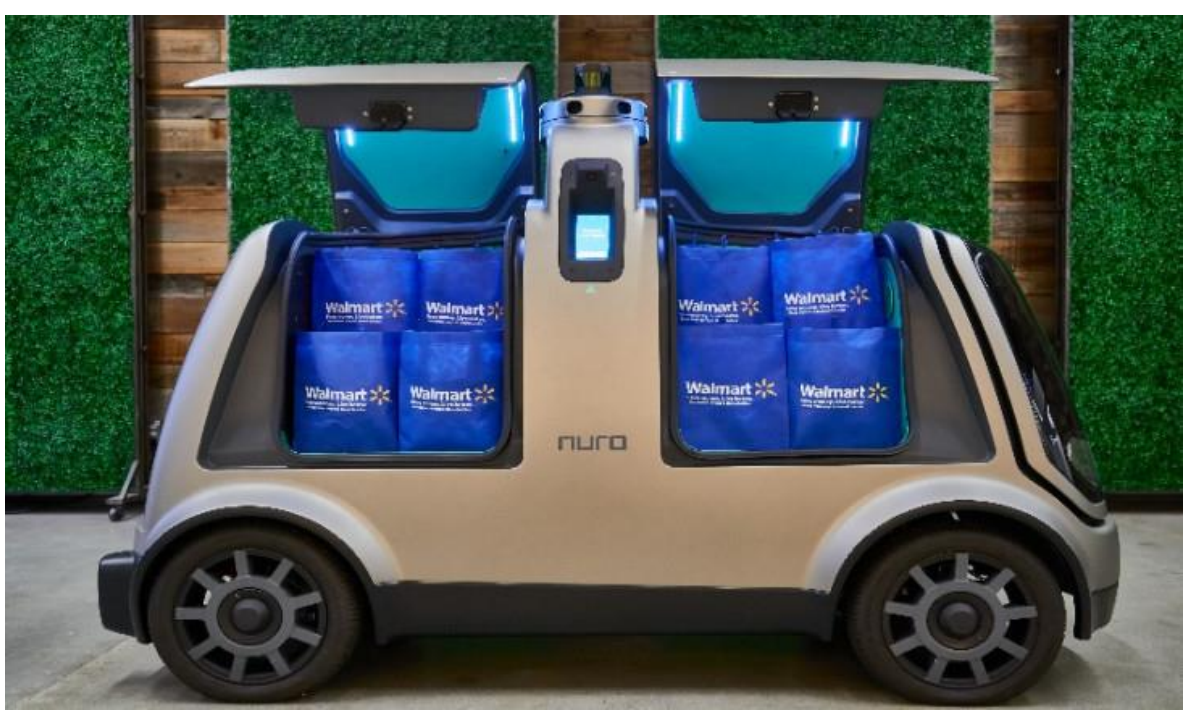

Figure 15 Nuro driverless vehicles. Credit: Nuro

The spread of coronavirus has put tremendous pressure on health systems worldwide. The virus has caused greater demand in laboratory testing as well as an urgent need for personal protective devices to allow patients with milder symptoms to be monitored remotely. Drone distribution is a valuable option as drones can easily carry samples from hospitals to labs, provide simple security services, and bring medical equipment to patients' homes such that immediate control can be provided. Currently some companies in China and Ghana are actively using this technology.

\section{Virtual Healthcare Assistants and Chatbots}

Since the beginning of the pandemic, many health care providers started to reduce in-person visits for a variety of patients by providing consultations over the phone or by video conference as a way to protect both patients and staff and to stop the spread of COVID-19. Information and communication technologies (ICTs) have great potential to address some of the challenges in this domain by providing accessible, cost-effective, and high-quality health care services. The World Health Organization (WHO) recommends its member states to take steps towards developing and implementing ICT based innovative solutions to reduce the need for personal contact between medical professionals and patients, and to increase accessibility to health care services [23]. Virtual healthcare assistants and chatbots (VHAC) are considered as one of the ICTs variants that aim to improve the conversation between healthcare providers and patients and put more information in the hands of the end users, to help healthcare organizations improve processes and reduce costs. VHAC are AI and rule-based systems that interact with humans to perform various tasks, to name a few, banking, answering phone calls, performing data entry, etc. These assistants use cognitive technologies such as machine learning, natural language processing (NLP), and neural networks to enable interactive communications with the end-users. Virtual assistant technology in the healthcare industry can assist in transforming various health processes and improve healthcare delivery worldwide. It helps improving healthcare quality, patient care, patient safety and patient outcome at lower costs [24]. VHAC can be considered as the optimum tool to provide healthcare services to vulnerable groups such as the elderly with low immunity and comorbidity. Recent reports claim that the global healthcare virtual assistant market is expected to grow at a rate of $24.7 \%$ from 2018 to 2025 to reach $\$ 1.7$ billion by 2025 [24]. 
With the increasing burden of communicable diseases, it is highly recommended to utilize technological advancement to control and monitor critical situations and decrease workload of healthcare workers. Technologies can facilitate receiving health care services without the need of unnecessary visits to the doctors [25]. It is believed that technological devices and ICT can be a great substitute for scarce human resources in this domain in the years to come [25]. On the daily life basis, virtual assistants can help providing reminders and motivational comments for medications, food and exercises [26]. They can perform regular checkups and answer questions regarding health issues [26]. They can be customized according to each person's interests and values in order to encourage people to participate in social interactions and community activities [26]. They can also play a role as a mediator among multiple experts from different fields [26]. The use of this technology to provide online and real-time healthcare services includes teleassessment, tele-diagnosis, tele-interactions and tele-monitoring [26]. For instance, telemonitoring (or remote monitoring) records data obtained from specific sensors, sends the data to specialists, and assists in decision making. The specialists can then make decisions based on other datasets and the patient's medical history [26].

Virtual assistants have been employed as a communicative agent. Researchers report that the usage of telemedicine increased between 2005 to 2017. The use of this technology improved rapidly for primary care telemedicine in 2016 and 2017 [27]. Consistently, based on a review study, the popularity of using individualized chat based system to deliver psychological therapies is increasing, although there are some deficiencies in methodological procedures [28-29]. There is a strong demand of more robust and efficient methods and standards for evaluating these technologies [29].

The need for implementing assistive technologies to fight COVID-19 has increased. It is reported Chinese health specialists are considering to use virtual technologies to provide consultation to patients [30]. During this difficult time, the Centers for Medicare \& Medicaid Services (CMS) has permitted people to use interactive applications to visit virtually their physician for a variety of purposes [30]. Canada, the UK, Italy, South Africa and India are some other countries that have deployed virtual health services[30].

Researchers, however, suggest further enhancing the capabilities of technology. "Virtual clinics" enables virtual visits with imaging data (e.g., chest X ray) to be remotely examined [31]. Regarding the merits of this approach, it is expected that it can help establishing equality among all people to receive a comprehensive examination and evaluation as well as reducing the number of patients in clinics and healthcare centers [30]. Furthermore, this could reduce the burden of healthcare workers [31].

In addition, virtual healthcare assistants can play a major role in preventive measures. "Chatbot" can help screen or identify early symptoms, teach and train users essential hygienic practices such as handwashing and safe grocery shopping [31]. Examples of the chatbots developed for COVID-19 are "Amplify.ai", and "Facebook's Messenger for COVID-19 Program". They provide information regarding the coronavirus and the COVID-19 pandemic [31]. Amplify.ai aims to create "interactive messenger experiences with support for both tapped and typed responses via advanced Natural Language Processing (NLP)" [32]. Another chatbot designed specifically for COVID-19 is "the Coronavirus Self-Checker" for those who perhaps are sick [33]. This chatbot provides resources to protect people and advices users whether or not they need to visit medical care centers. However, this system is designed to provide information only to Americans [33]. 
During the fight against the pandemic, voice assistants are also widely used. A voice assistant can complete 200 calls in 5 minutes and produce statistical results. Manual calls, on the other hand, requires 2 to $3 \mathrm{~min}$ per call, plus the statistical analysis that requires at least 3 to 4 hours for processing. With the trial of voice robots, the efficiency of epidemic investigation has been greatly improved. During the pilot process, a voice assistant will automatically call the recipient's phone, and multiple rounds of interactive conversations with the recipient can be performed to collect and confirm the information, including identity information, areas visited, recent contact groups, and current symptoms. After confirming the information, the voice assistant will automatically classify the statistics and promptly provide daily statistical results [16].

\section{Drug Repurposing/Discovery and Vaccination Discovery}

AI can mimic the human decision-making process. Machines can learn from data and human expertise. Science reports the most suitable tasks for AI include: learning a function that maps well-defined inputs to well-defined outputs, large data sets exist or can be created containing inputoutput pairs, the task provides clear feedback with clearly definable goals and metrics, no long chains of logic or reasoning that depend on diverse background knowledge or common sense, no need for detailed explanation of how the decision was made, a tolerance for error and no need for provably correct or optimal solutions, the phenomenon or function being learned should not change rapidly over time, no specialized dexterity, physical skills or mobility required. The unique properties of AI make it suitable for solving various medical problems, such as drug repositioning and vaccination discovery.

The United Nations has been encouraging nations to develop technologies for advanced health diagnostics through AI and big data, enhanced access to health care through telemedicine, blockchain and AI optimized medical data. AI can improve infectious disease monitoring, identify potential risks. AI has been used to classify bat species likely to host filovirus that causes severe fevers like Ebola. The developed model achieved 87\% accuracy, which helped improve countries' ability to handle outbreaks. AI may surpass medical experts in diagnosis, analysis, and analytics for drug development. Analytics models and data-sharing tools developed by DeepMind have been utilized to identify deterioration by public health services in the UK and US.

Traditionally, the solution to dangerous new bacterial and viral infections is to create a mixture of different medicines. However, with the drug combinations sub-optimally selected and doses selected through trial and error, this procedure is laborious and time-consuming. This expensive and inefficient approach to create a cure is complicated when a fast response to the global pandemic is necessary and resources need to be maintained.

Conventional diagnosis collection involves the analysis of the virus or the development of bacteria in reaction to various potential drugs. Drugs are administered in growing doses to the bacteria or viruses before optimum growth suppression is detected. To intensify the effect, additional medicines are added together. But when many medications are tested concurrently as options, these approaches are not always possible. These methods often yield promising effects but are not verified through human trials [34, 35].

The effects of all potential drug formulations and the optimal mixture of drugs are difficult to validate using conventional approaches. Moreover, if a drug from a list of candidate drugs has not 
shown a significant impact on a pathogen, it typically is no longer included in the conventional sampling.

Professor Ho and his staff, along with colleagues from Shanghai Jiao Tong University, have applied AI for drug development. The vesicular stomatitis virus (VSV) infection was deliberately picked as 12 suitable candidates for diagnosis. Their development also reduced the number of tests available to evaluate the full spectrum of formulations and appropriate doses of these 12 drugs with IDentif. AI [34]. "This time and precision in the discovery of new drug combination therapy is somewhat unparalleled", as Professor Ho states, "We spent three days to identify multiple optimized medication schematics from thousands of possible combinations which reduced the VSV infection to $1.5 \%$ without obvious negative consequences." Most notably, the team has seen the seven-fold higher effectiveness than sub-optimal doses in the maximum dosage for the topranking drug mix. This shows that ideal dose detection is essential. Similarly, the mixture was 14 times less successful when a single medication was supplemented by the medication mix and delivered at sub-optimal doses.

The results of IDentif. AI prove it's critical to think about how the medicine develops and is subsequently given in a combination [34]. They concluded that techniques like IDentif. AI, which can easily automate pharmaceutical use during austere economic conditions in the midst of pandemics, would lead to improved medical outcomes compared to traditional approaches. The team is now looking at COVID-19, having proven the efficacy of IDentif. AI in providing care for infectious diseases. Professor Ho said, "We will need a fast-clinical approach to combat VICs that can evolve over time because of the current production of vaccines and antibody therapies for COVID-19. Our strength is that we can run one trial and come up with a list of drug combinations for therapy within days. Prof. Ho concluded, "When a disease enters an active outbreak, it would be important for a quick response and this solution will continue to change as the pathogen evolves [34].

Various businesses and start-ups have innovatively changed their work goals in order to use AI to speed up the quest for a cure against COVID-19. To help researchers easily synthesize and test new candidate molecules against COVID-19, the European IA-centric starting Molecule.one has laid down a proprietary synthesis method for open access to the scientific Community. IBM's AI frameworks have been used to cover three COVID-19 targets, and 3000 novel molecules have been developed. Such molecules were published to the science community for synthesis, processing and optimization, under the Creative Commons License (CCL). A list of 97 small molecules candidates intended to inhibit the SARS-CoV-2 3CL protease has been published by InSilico Pharmacy, a Hong Kong based drug development company. A variety of fast virtual screening AI models with existing public and commercial compound libraries as key repositories have been developed and evaluated against COVID-19, respectively. Essentially, numerous research avenues have combined AI models for future therapeutics to be produced at an unparalleled speed for COVID-19.

To date, both the bases and the proliferation of the COVID19 pathogenesis are still unclear, and no vaccine nor proven treatment has yet been discovered. As a fast-acting solution, in silico computational methods can be utilized for discovery and even designing of novel drugs [35]. The short-time requirements of computational methods are productive for high throughput screening of the currently known drugs to define the significant drugs for newly emerging diseases, besides the prediction of the negative impacts of novel drugs [36]. On the contrary, the development of novel drugs via clinical approval requires several years of work, which is a time-consuming 
process [37]. However, drug repositioning, also known as repurposing, is a potential strategy to control the emerging diseases caused by infectious agents that are vastly spreading [38]. Moreover, drugs that have been approved for a closely linked disease or even sharing the same symptoms and/or complications, are safe for human use, but only their efficacy against the COVID-19 is required to be re-examined, validated and established [39]. In life-threatening cases, where there is no alternative medicine or vaccine, such a drug repurposing strategy is particularly attractive. However, clinical trials are essential to ensure that such proposed treatment is acting better than a placebo [40]. Lopinavir and Ritonavir were mentioned in earlier literature to target the Mpro of SARS virus. The protein sequences of COVID-19 Main protease (2019-nCoV Mpro) and SARSCoV Mpro are $96 \%$ identical [41]. In several early studies, the similarities in the sequence of a potential target for COVID-19 to that of the SARS Mpro were utilized to build a model for the structure of SARSCoV-2 Mpro [41]. Homology based models were utilized to screen a library of compounds to predict that Nelfinavir, an approved antiviral protease inhibitor, is a potential drug for COVID-19 [42]. The sequence similarity of the SARS-CoV-2 Mpro to the SARS Mpro is sufficiently high to build a good model for the structure of SARS-CoV-2 Mpro [38, 43]. However, the predictions of virtual screening studies and binding energy calculations are generally more accurate if a high-resolution experimental structure of the target is available.

Methods based on AI are emerging as exciting methods for exploration of the enormous chemical space for sampling drug-like molecules. Models of AIs will test characteristics based on current medical devices that can be used in the search for more pharmaceuticals in the chemical area. The product design group has been able to tackle a variety of issues, including the current antibiotic resistance epidemic. Most notably, an AI strategy will minimize from many years to a few days the initial process of medicament exploration.

\section{Ethical Implication of AI and Robotics}

Previous sections highlighted how important Artificial Intelligence and Robotics are for addressing COVID-19. At same time these domains can bring a lot of benefits for the humanity, they can pose serious risks for our fundamental rights ranging from the impact on democracy to our right to life.

Currently, several Governments are establishing partnerships with private companies to get access and track people's mobile. In [44], one can see a live index elaborated by Top10PVN firm, about the use of mobile location per country which ranges from processing anonymized data to the tracking of an individual person and her contacts. Governments argue mobile location can provide invaluable information to help understanding the dynamic of COVID-19 and, thus, elaborating public policies. In fact, the data collected can be analyzed to identify patterns of movements which can be used together with data on testing and diagnosis to inform how the virus is being spread in a particular region or, even, at individual level, alert authorities if a particular individual is obeying a home quarantine [45].

The main concern in these efforts is they can open the door to a further and wider use of this kind technology to other domains increasing the surveillance of the citizens under different and questionable justifications. This fact raises several concerns about potential misuses that directly would impact our privacy, freedom of expression and freedom of association accentuating, e.g., discrimination and further harming marginalized groups. Recently, a joint statement [46] signed by different organizations urged Governments ensure the use of digital technologies to address the pandemic is in line with human rights. In this statement, the signatories listed several conditions 
Governments should observe that include all surveillance measures must be lawful, necessary and proportionate and all monitoring and surveillance powers must be time-bound, and only continue for as long as necessary to address the current pandemic. Considering the latter, Amnesty International [47], emphasizes "there is a real danger surveillance measures become permanent fixtures. In the wake of the attacks of 11 September 2001 (9/11), government surveillance apparatus expanded significantly. Once these capabilities and infrastructure are in place, governments seldom have the political will to roll them back". Thus, what guarantees we have that companies and Government will stop the surveillance or even discard all collected and processed data once the pandemic ends?

Another relevant issue is if an AI-based system should ultimately decide who should live or not, or simply if someone should receive or not a medical treatment. This debate is not new, but it has increased those days during the Pandemic. The world was not prepared for a Pandemic and some countries are facing some life and death dilemmas due to resources limitation. The question is what are the data should be used to provide an answer? Should age, gender, known diseases, race, social status or other be considered? How should all these components be combined and why? In China, some researchers developed an AI-based tool to assist doctors to make choices about patient with COVID-19 using blood samples [48]. Systems that provide support to human decisions are very welcome. However, they should never undermine human agency, i.e., humans should not be obligated to use system decisions blindly without having them scrutinised. The reason is simple. AI-based systems are just math tools. They are neither intelligent nor neutral. They are fairly as good as the quality of the data that is used in their training set. This fact is corroborated with evidences presented in different reports that show gross errors produced by some systems, which a human would never make [49].

A correlated problem is most of the people that will use AI-based systems does not know their limitations. This has led to situations where some people in key positions anthropomorphize and over-trust the technology, and using fallacious arguments, impose the adoption of these systems in critical and sensitive tasks. Therefore, initiatives like IEEE Global Initiative on Ethics of Autonomous and Intelligent Systems [50] and IEEE/MIT Global Council on Extended Intelligence [51] are very relevant. While the former aims to educate and empower people to take ethics into consideration during the life cycle of AI-based systems, the latter tries to shed some lights on AI creating a new narrative for the domain to demystify the hype that surrounds it.

The risk for misusing technology is high. Organizations like ProPublica recently pointed out people with disabilities may be denied from lifesaving care during the Pandemic [52]. It cites some disasters preparedness plans in which people with cognitive issues have lower priority for lifesaving treatment. In Brazil, the Ministry of Health suggested in a press collective [53] that saving an elderly is more financially expensive than saving a young. As the young has an entire life in front of her, she would have preference to be saved. Information like those can be incorporated in an AI-based system causing irreparable losses, and due to the opaqueness of the system, this discriminatory information would never be uncovered and the responsible would never be punished.

\section{Conclusion}

The global battle against novel coronavirus and COVID-19 pandemic has seen AI and robotics play extremely important roles in understanding the scope of the pandemic, dealing with the contagious nature of the virus and decelerating its spread. This chapter 
reviewed a number of $\mathrm{AI}$ and robotics applications in the battle against COVID-19. These applications include early detection and diagnosis, massive agent modeling and simulation, data analytics, assistive robots, disinfection robots, public awareness and patrolling, contactless delivery services, chatbots for medical care and drug repurposing and vaccination discovery. Ethical implications of AI and robotics were also reviewed.

Many lessons are learnt from this unprecedented crisis that has profoundly impacted human capital, including lives, well-being, learning, and productivity. There is a need to fully understand the limitations of AI-based systems for not over-trusting the technology and creating false arguments especially in critical health-related tasks. Measures should be taken to protect digital rights, stop the surveillance once the pandemic ends, ban new totalitarianism of surveillance technology and digital totalitarian states. Robotics and AI in general have to be regulated and smartly directed toward the benefit of humanity.

\section{References}

[1] Yang, Guang-Zhong, Bradley J. Nelson, Robin R. Murphy, Howie Choset, Henrik Christensen, Steven H. Collins, Paolo Dario et al. "Combating COVID-19-The role of robotics in managing public health and infectious diseases." Science robotics, 25 Mar 2020: Vol. 5, Issue 40, DOI:

10.1126/scirobotics.abb5589 (2020).

[2] Alaa Khamis, "Biological versus Non-Biological/Artificial Intelligence", Medium, March 2019, available at: https://medium.com/@alaakhamis/biological-versus-non-biological-artificial-intelligence9dee32a1517d, last accessed: May 2, 2020.

[3] Wu Fan. Epidemic promotes surge in demand for infrared temperature measuring instruments: we "reunite families" and they "overtime"[N]. Science and Technology Board Daily. 2020.02.03.

[4] Shenzhen Municipal Affairs Service Data Administration. Epidemic Pass Reference Architecture and Technical Guide. 2020.03.05.

[5] QiRu Sun. AR glasses check the car, induction disinfection, Zhongguancun resumed work using these high-tech[N]. Beijing Daily. 2020.02.28.

[6] Li Lin, Qin Lixin, Xu Zeguo. Artificial Intelligence Distinguishes COVID-19 from Community Acquired Pneumonia on Chest CT[J]. Radiology. 2020.03.19.

[7] Wang, Y.,Hu, M., Li, Q., Zhang, X. P., Zhai, G., \& Yao, N. (2020). Abnormal respiratory patterns classifier may contribute to large-scale screening of people infected with COVID-19 in an accurate and unobtrusive manner. arXiv preprint arXiv:2002.05534.

[8] Alaa Khamis, Turning Data into Actionable Insights, Medium, March 2019, available at:https://medium.com/@alaakhamis/turning-data-into-actionable-insights-c246969fa4c, last accessed: May 2, 2020.

[9] Wu, Joseph T., Kathy Leung, and Gabriel M. Leung. "Nowcasting and forecasting the potential domestic and international spread of the 2019-nCoV outbreak originating in Wuhan, China: a modelling study." The Lancet 395.10225 (2020): 689-697.

[10] Kucharski, Adam J., et al. "Early dynamics of transmission and control of COVID-19: a mathematical modelling study." The lancet infectious diseases (2020). 
[11] James Gallagher, "Coronavirus: When will the outbreak end and life get back to normal?", BBC, March 23, 2020, last accessed: April 25, 2020.

[12] Pueyo, Tomas. "Coronavirus: The hammer and the dance." Available at: "https://medium"https://medium. com/@ tomaspueyo/coronavirus-the-hammer-and-the-dancebe9337092b56, last accessed: April 25, 2020.

[13] Sun Han, Yang Fan. Discussion on the Application of Logistics Robot Distribution in Community Epidemic Isolation[J]. Digital Communication World. 2020.02.

[14] Robert F. Service, "Does disinfecting surfaces really prevent the spread of coronavirus?". Science Magazine, March 12, 2020, doi:10.1126/science.abb7058.

[15] Shadrin, Gennady K., et al. "Application of Compensation Algorithms to Control the Movement of a Robot Manipulator." Acta Polytechnica Hungarica 17.1 (2020).

[16] Námesztovszki, Zsolt, et al. "The Correlations between Health Behaviors and the Use of HealthPreserving Mobile Applications in Young Adults." Acta Polytechnica Hungarica 17.2 (2020).

[17] R Goodman. 2005. Whatever you call it, just don't think of last-mile logistics, last. Global Logistics \& Supply Chain Strategies 9, 12 (2005), 46-51.

[18] Martin Joerss, Jürgen Schröder, Florian Neuhaus, Christoph Klink, and Florian Mann. 2016. Parcel delivery: The future of last mile. McKinsey \& Company (2016).

[19] T. Niels, M. T. Hof, and K. Bogenberger. 2018. Design and operation of an urban electric courier cargo bike system. In 2018 21st international conference on intelligent transportation systems (itsc). 2531-2537. https://doi.org/10.1109/ITSC.2018.8569606

[20] Li Nianzhen. How a Chinese drone delivery startup is capitalizing on COVID-19. Retrieved April 18,20 from https://asia.nikkei.com/Business/Startups/How-a-Chinese-drone-delivery-startup-iscapitalizing-on-COVID-19

[21] Bangkok Post. Robot to deliver meals, medication to Covid-19 patients in S'pore9. Retrieved April 18,20 from https://www.bangkokpost.com/world/1872349/robot-to-deliver-meals-medication-to-covid19-patients-in-spore

[22] Steve Crowe. Nuro driverless vehicles approved for delivery tests in California. Retrieved April 18,20 from https://www.therobotreport.com/nurodriverless-delivery-vehicles-approved-california/

[23] World Health Organization (WHO) (2009). Telemedicine: opportunities and developments in Member States: report on the second global survey on eHealth. ISBN: 978-92-4-156414-4. Available online PDF at: http://www.who.int/goe/publications/goe_telemedicine_2010.pdf

[24] Healthcare Virtual Assistant Market - Global Opportunity Analysis And Industry Forecast (20182025). Retrived May 9, 2020 from https://www.meticulousresearch.com/product/healthcare-virtualassistant-market/.

[25] Breen, G.-M., \& Matusitz, J. (2010). An Evolutionary Examination of Telemedicine: A Health and Computer-Mediated Communication Perspective. Social Work in Public Health, 25(1), 59-71. https://doi.org/10.1080/19371910902911206 
[26] Kuziemsky, C., Maeder, A. J., John, O., Gogia, S. B., Basu, A., Meher, S., \& Ito, M. (2019). Role of Artificial Intelligence within the Telehealth Domain. Yearbook of Medical Informatics, 28(1), 35-40. https://doi.org/10.1055/s-0039-1677897

[27] Barnett, M. L., Ray, K. N., Souza, J., \& Mehrotra, A. (2018). Trends in Telemedicine Use in a Large Commercially Insured Population, 2005-2017. JAMA, 320(20), 2147-2149.

https://doi.org/10.1001/jama.2018.12354

[28] Hoermann, S., McCabe, K. L., Milne, D. N., \& Calvo, R. A. (2017). Application of Synchronous Text-Based Dialogue Systems in Mental Health Interventions: Systematic Review. Journal of Medical Internet Research, 19(8). https://doi.org/10.2196/jmir.7023

[29] Laranjo, L., Dunn, A. G., Tong, H. L., Kocaballi, A. B., Chen, J., Bashir, R., Surian, D., Gallego, B., Magrabi, F., Lau, A. Y. S., \& Coiera, E. (2018). Conversational agents in healthcare: A systematic review. Journal of the American Medical Informatics Association : JAMIA, 25(9), 1248-1258. https://doi.org/10.1093/jamia/ocy072

[30] Webster, P. (2020). Virtual health care in the era of COVID-19. The Lancet, 395(10231), 11801181. https://doi.org/10.1016/S0140-6736(20)30818-7

[31] Ting, D. S. W., Carin, L., Dzau, V., \& Wong, T. Y. (2020). Digital technology and COVID-19. Nature Medicine, 26(4), 459-461. https://doi.org/10.1038/s41591-020-0824-5

[32] Covid19 - Amplify.ai. (2020). Retrieved May 8, 2020, from http://amplify.ai/covid19/

[33] COVID-19 Risk Screening Tool. (2020). Retrieved May 8, 2020, from https://covid19chat.clevelandclinic.org/

[34] Ho, D. (2020), Addressing COVID-19 Drug Development with Artificial Intelligence. Adv. Intell. Syst.. doi:10.1002/aisy.202000070.

[35] Macalino, S.J.Y., Gosu, V., Hong, S. and Choi, S. (2015) Role of computer-aided drug design in modern drug discovery. Archives of pharmacal research, 38(9), pp. 1686-1701.

[36] Talluri, S. (2019) Computational Protein Design of Bacteriocins based on structural scaffold of aureocin A53. International Journal of Bioinformatics Research and Applications, 15(2), 129-143.

[37] Strømgaard, K., Krogsgaard-Larsen, P. and Madsen, U. Eds. (2017) Textbook of drug design and discovery. CRC press.

[38] Li J, Zheng S, Chen B, Butte AJ, Swamidass SJ, Lu Z. (2016) A survey of current. Trends in computational drug repositioning. Briefings in bioinformatics, 17(1):2-12.

[39] Hurle, M.R., Yang, L., Xie, Q., Rajpal, D.K., Sanseau, P. and Agarwal, P. (2013) Computational drug repositioning: from data to therapeutics. Clinical Pharmacology \& Therapeutics, 93(4), 335-341.

[40] Novac, N. (2013) Challenges and opportunities of drug repositioning. Trends in pharmacological sciences, 34(5), pp.267-272.

[41] Liu, X.; Zhang, B.; Jin, Z.; Yang, H.; Rao, Z. The crystal structure of 2019-nCoV main protease in complex with an inhibitor N3. PDB ID 6LU7 dated 12-Feb-2020.

[42] Xu, X., Jiang, X., Ma, C., Du, P., Li, X., Lv, S., \& Li, Y. (2020). Deep learning system to screen coronavirus disease 2019 pneumonia. arXiv preprint arXiv:2002.09334. 
[43] Chen, L.; Liu, W.; Zhang, Q.; Xu, K.; Ye, G.; Wu, W.; Sun, Z.; Liu, F.; Wu, K.; Zhong, B.; Mei, Y.; Zhang, W.; Chen, Y.; Li, Y.; Shi, M.; Lan, K. \& Liu, Y. (2020), 'RNA based mNGS approach identifies a novel human coronavirus from two individual pneumonia cases in 2019 Wuhan outbreak', Emerg Microbes Infect 9(1), 313-319.

[44] COVID-19 Digital Rights Tracker. Available at https://www.top10vpn.com/news/surveillance/covid19-digital-rights-tracker/

[45] Poland is making its citizens use a 'selfie' app during the coronavirus crisis.

https://www.abc.net.au/news/2020-04-25/coronavirus-poland-tracking-quarantine-selfie-app/12173884

[46] Joint Civil Society Statement: States use of digital surveillance technologies to fight pandemic must respect human rights. Available at https://www.hrw.org/news/2020/04/02/joint-civil-society-statementstates-use-digital-surveillance-technologies-fight

[47] COVID-19, surveillance and the threat to your rights. Available at https://www.amnesty.org/en/latest/news/2020/04/covid-19-surveillance-threat-to-your-rights/

[48] Should AI help make life-or-death decisions in the coronavirus fight? Available at https://www.scmp.com/news/china/science/article/3076259/should-ai-help-make-life-or-death-decisionscoronavirus-fight

[49] Why deep-learning AIs are so easy to fool Artificial-intelligence - Researchers are trying to fix the flaws of neural networks. Available at https://www.nature.com/articles/d41586-019-03013-5

[50] Ethics in Action. Available at https://ethicsinaction.ieee.org

[51] CXI - Council on Extended Intelligence. https://globalcxi.org

[52] People with Intellectual Disabilities May Be Denied Lifesaving Care Under These Plans as Coronavirus Spreads. Available at https://www.propublica.org/article/people-with-intellectualdisabilities-may-be-denied-lifesaving-care-under-these-plans-as-coronavirus-spreads?

[53] (In Portuguese) Teich já sugeriu que salvar idoso em vez de jovem é mais caro à Saúde. https://www.cartacapital.com.br/politica/teich-ja-sugeriu-que-salvar-idoso-em-vez-de-jovem-e-mais-caroa-saude/ 